\title{
NONTHERMAL PARTICLES AND RADIATION PRODUCED BY CLUSTER MERGER SHOCKS
}

\author{
Robert C. Berrington and Charles D. Dermer \\ Naval Research Laboratory, Code 7653, 4555 Overlook Avenue, SW, Washington, DC 20375-5352; \\ rberring@gamma.nrl.navy.mil, dermer@gamma.nrl.navy.mil \\ Received 2002 September 12; accepted 2003 May 12
}

\begin{abstract}
We have developed a numerical model for the temporal evolution of particle and photon spectra resulting from nonthermal processes at the shock fronts formed in merging clusters of galaxies. Fermi acceleration is approximated by injecting power-law distributions of particles during a merger event, subject to constraints on maximum particle energies. We consider synchrotron, bremsstrahlung, Compton, and Coulomb processes for the electrons, nuclear, photomeson, and Coulomb processes for the protons, and knock-on electron production during the merging process. The broadband radio through $\gamma$-ray emission radiated by nonthermal protons and primary and secondary electrons is calculated both during and after the merger event. Using ROSAT observations to establish typical parameters for the matter density profile of clusters of galaxies, we find that typical merger shocks are weak and accelerate particles with relatively soft spectra. We consider the prospects for detecting nonthermal radio and $\gamma$-ray emission from clusters of galaxies and implications for the origin of ultra-high-energy cosmic rays and the diffuse $\gamma$-ray background. Our results suggest that only a few of the isotropically distributed unidentified EGRET sources are due to shocks formed in cluster mergers and that only a minor contribution to the diffuse extragalactic $\gamma$-ray background can originate from cluster merger shocks. Cluster merger shocks can accelerate protons to $\lesssim 10^{19} \mathrm{eV}$ for the standard parameters considered here. We predict that GLAST will detect several cluster mergers, and depending on the mean magnetic fields in the intracluster medium, the Low Frequency Array could detect anywhere from several to several hundred.
\end{abstract}

Subject headings: acceleration of particles — galaxies: clusters: general — methods: numerical — shock waves - X-rays: galaxies: clusters

\section{INTRODUCTION}

According to the hierarchical merging scenario, cold dark matter halos evolve to form larger structures by merging with adjacent dark matter halos. Within dark matter halos, baryonic matter condenses to form clusters of galaxies. A cluster merger event results from the interaction of galaxy clusters during the merger of cold dark matter halos. The gravitational potential energy available in a cluster merger event involving halos with masses $\sim 10^{15} M_{\odot}$ is $\sim 10^{63}-10^{64}$ ergs. As two clusters of galaxies merge, the infall velocities can exceed the sound speed of the intracluster medium (ICM). As a result, a shock front will form at the interaction boundary between the clusters. First-order Fermi acceleration at the shock front produces a population of nonthermal, relativistic particles. Relativistic electrons are detected from their synchrotron radio emission or from $\gamma$-rays due to Compton-scattered cosmic microwave radiation. Nonthermal protons are detected through $\gamma$-rays emitted from secondaries formed in nuclear production processes, including the $\pi^{0}$-decay signature at $70 \mathrm{MeV}$.

Rich clusters contain a hot and tenuous ionized ICM, with observed temperatures $T_{\mathrm{X}} \approx 5-10 \mathrm{keV}$, sound speeds $\sim 1000 \mathrm{~km} \mathrm{~s}^{-1}$, and thermal bremsstrahlung luminosities $L_{\mathrm{X}} \sim 10^{45} \mathrm{ergs} \mathrm{s}^{-1}$ between 2 and $10 \mathrm{keV}$. In addition to the luminous thermal component present in these clusters, there is a growing body of evidence supporting the presence of nonthermal distributions of particles in cluster mergers (Eilek 1999; Feretti et al. 2000). Deep radio observations of clusters of galaxies indicate the presence of extended diffuse emission not easily associated with an optical counterpart. These diffuse radio features are commonly classified as either radio halos or radio relics (which are also called periphery halos). Radio halos mimic the observed X-ray profiles and are characterized by their central location in the cluster and by a highly disorganized magnetic field and are generally thought to be a consequence of a merger event (Feretti et al. 2000). Radio relics, usually found on the periphery of the cluster, are characterized by highly organized magnetic fields and often display filamentary structures. We focus here on radio relics, which are thought to result from synchrotron emission emitted by electrons directly accelerated at shock fronts. By contrast, radio halos might be due to reacceleration of relic electrons by magnetic turbulence or enhanced magnetic turbulence arising from the cluster merger or motions of the galaxies (Brunetti et al. 2001; Ohno, Takizawa, \& Shibata 2002).

The first radio halo in a cluster of galaxies was detected from the Coma Cluster (Large, Mathewson, \& Haslam 1959). Many halos and relics have since been found in a number of other clusters, including A754 (Kassim et al. 2001), A2256 (Bridle \& Fomalont 1976), and others (see Govoni et al. 2001; Slee et al. 2001 for recent observations). Typical radio powers from the radio halos and relics are at a level of $10^{40}-10^{42} \mathrm{ergs} \mathrm{s}^{-1}$ (Giovannini \& Feretti 2000). These clusters show a favorable correlation (Kassim et al. 2001; Berrington, Lugger, \& Cohn 2002) between the existence of diffuse radio features and recent or ongoing merger activity. Detection of these features is largely due to sensitivity limitations; the number of detected diffuse radio features has increased with the improvement in sensitivity of radio telescopes.

The synchrotron evidence for nonthermal electrons in clusters of galaxies indicates that an acceleration mechanism must exist in the environment of the host cluster with sufficient power to produce the observed emission. Several 
mechanisms have been proposed to explain the correlation of the radio halo and relic features and the recent or ongoing merger activity. These mechanisms often require the presence of shock waves or magnetic turbulence to accelerate particles via the Fermi acceleration process (Schlickeiser, Sievers, \& Thiemann 1987; Tribble 1993; Kang, Rachen, \& Biermann 1997; Ensslin et al. 1998; Blasi 2000; Miniati et al. 2001). Other theories include adiabatic compression of fossil radio plasma by a cluster merger shock wave (Ensslin \& Gopal-Krishna 2001).

Optical surveys show that approximately $30 \%-40 \%$ of clusters of galaxies display evidence for the presence of substructure (Forman et al. 1981; Geller \& Beers 1982 and others). This internal structure is often interpreted as a subset of galaxies merging with a larger cluster of galaxies. These internal structures indicate velocity differences near or greater than the expected sound speed of the ICM. Observed velocities typically range from $\sim 1000$ to $3000 \mathrm{~km}$ $\mathrm{s}^{-1}$, which are consistent with values expected from parabolic orbits (Oegerle \& Hill 1994).

Emission from nonthermal particles will also appear at EUV and hard X-ray (HXR) energies as a power-law excess. While the EUV emission seen in the Coma Cluster (Lieu et al. 1999b) and possibly also A2199 and A1795 (Lieu, Bonamente, \& Mittaz 1999a) may have a cool thermal origin, it is unlikely because of the extreme mass requirements of cool gas. It is more likely that the EUV emission has a nonthermal origin (Hwang 1997; Ensslin \& Biermann 1998; Mittaz, Lieu, \& Lockman 1998; Blasi \& Colafrancesco 1999; Atoyan \& Völk 2000). Excess HXR emission has been reported from the clusters A1656 (Fusco-Femiano et al. 1999; Rephaeli, Gruber, \& Blanco 1999), A2256 (Fusco-Femiano et al. 2000), A3667 (Fusco-Femiano et al. 2001), and possibly A2199 (Kaastra et al. 1999). Estimated luminosity of the HXR emission is $\approx 10^{43} \mathrm{ergs} \mathrm{s}^{-1}$. Thermal origins of this HXR excess emission would require unrealistic temperatures of greater than $40 \mathrm{keV}$, and so it is thought to be caused by the presence of relativistic nonthermal electrons (Fusco-Femiano et al. 1999).

Numerical models of merging clusters of galaxies (Ricker 1998; Takizawa 2000; Ricker \& Sarazin 2001; Miniati et al. 2001 and others) have treated the development of shocks as a result of the cluster merging process. Given the presence of thermal ionized particles in the vicinity of these shocks and a cluster magnetic field, electrons and ions will be accelerated via the first-order Fermi process (Bell 1978; Blandford \& Ostriker 1978). Numerous attempts have been made to model the emissions from nonthermal particles (Colafrancesco \& Blasi 1998; Fujita \& Sarazin 2001; Petrosian 2001; Miniati et al. 2001) produced by these cluster merger shocks, but this study differs from previous attempts in that we accurately model the diffusion of particles in energy space due to Coulomb interactions and allow for a variable injection rate that depends on the environment local to the shock front. Recent studies by Liang, Dogiel, \& Birkinshaw (2002) have addressed the balance of Coulomb losses and diffusion in energy space but do not address the injection and diffusion of particles in energy space along with a variable source function of nonthermal particles. In our treatment, we follow particle energies up to $10^{19} \mathrm{eV}$ and calculate the bremsstrahlung, Compton, synchrotron, and $\pi_{0} \gamma$-rays from $p-p$ collisions. Because the shock front lifetime is a significant fraction of the age of the universe, we also follow the changing environment due, for example, to the changing cosmic microwave background (CMB) energy density. In addition, we accurately model nonthermal electrons and protons up to $\sim 10^{21} \mathrm{eV}$, although we find that limitations on particle acceleration make it difficult to produce protons above $\sim 10^{19} \mathrm{eV}$ in cluster merger shocks. From the particle distributions, we calculate nonthermal photon spectra for energies up to $\sim 10^{7} \mathrm{GeV}$. We also include the effects of secondary production on the nonthermal photon spectra.

The physical processes and the temporal evolution of the particles are described in $\S 2$. The results of the simulations are presented in $\S 3$. A comparison of the photon spectra with observed clusters and the potential of detecting these shocks with space-based satellite observatories is discussed in $\S 4$.

\section{MODELS}

We have developed a code to calculate the time-dependent particle distribution functions evolving through radiative losses for electrons and protons accelerated by the firstorder Fermi process at the cluster merger shock. The code was originally adapted from a supernova remnant code (Sturner et al. 1997) and applied to the specific case of the cluster merger scenario. We break up the discussion of the problem into four subsections: cluster merger dynamics, nonthermal particle production, temporal evolution, and the production of the photons.

\subsection{Cluster Merger Dynamics \\ 2.1.1. Evolution of the Merger Event}

Two clusters are assumed to be the dominant gravitating masses in the local region, and variations of the gravitational field due to surrounding masses are assumed to be negligible. A cluster accretes another cluster that falls from a distance $d$. This distance can be approximated by the maximum separation of two point masses that move apart beginning at the moment of the big bang, follow an elliptical orbit, and merge at time $t_{m}$. Given this assumption, a cluster of mass $M_{1}=10^{15} M_{15} M_{\odot}$ accretes a subcluster of mass $M_{2}=10^{15} m_{15} M_{\odot}$ at time $t_{m}$, as approximated by Kepler's third law. Consequently, a cluster that merges at a time $t_{m}$ fell from a maximum separation approximated by

$$
\begin{aligned}
d & \approx\left[\frac{2 G\left(M_{1}+M_{2}\right) t_{m}^{2}}{\pi^{2}}\right]^{1 / 3} \\
& \approx 4.5\left(\frac{M_{1}+M_{2}}{10^{15} M_{\odot}}\right)^{1 / 3}\left(\frac{t_{m}}{10^{10} \mathrm{yr}}\right)^{2 / 3} \mathrm{Mpc}
\end{aligned}
$$

(Ricker \& Sarazin 2001). This equation allows us to calculate the magnitude of the energy pool available for particle acceleration. The total orbital energy $\mathscr{E}$ of the merging system, assuming a zero impact parameter, is given by

$$
\begin{aligned}
\mathscr{E} & \approx-\frac{M_{1} M_{2}}{2^{1 / 3}\left(M_{1}+M_{2}\right)^{1 / 3}}\left(\frac{\pi G}{t_{m}}\right)^{2 / 3} \\
& \approx-1.9 \times 10^{64} \frac{M_{15} m_{15}}{\left(M_{15}+m_{15}\right)^{1 / 3}}\left(\frac{t_{m}}{10^{10} \mathrm{yr}}\right)^{-2 / 3} \mathrm{ergs} .
\end{aligned}
$$

We use the terminology that orbits are bound if the total energy $\mathscr{E}<0$; otherwise, they are unbound. It is customary to use the Keplerian analogy of elliptical to represent orbits where $\mathscr{E}<0$ and parabolic for orbits where $\mathscr{E}=0$. 
To approximate the merger velocity of the system, we relax the assumption that the accreting cluster is a point mass. Instead, we assume the ICM number density of the accreting cluster follows the spherically symmetric, isothermal beta model given by

$$
n_{\mathrm{ICM}}(r)=n_{0}\left[1+\left(\frac{r}{r_{c}}\right)^{2}\right]^{-3 \beta / 2} .
$$

The quantity $n_{0}$ is the central number density of the ICM, and $r_{c}$ is the core radius, which characterizes the spatial scale over which the density changes from a roughly constant value at $r \lesssim r_{c}$ to a power-law density dependence at $r \gtrsim r_{c}$. The gravitational dynamics are dominated by the dark matter distribution of the more massive cluster. Assuming that the dark matter distribution follows a profile similar to that of the ICM and that the merging cluster is a point mass, we calculate the cluster merger velocity and radial separation by solving the differential equation

$$
m_{r} \frac{d^{2} r_{12}}{d t^{2}}=-M_{2} \frac{d \Phi\left[M_{1}\left(r_{12}\right), r_{12}\right]}{d r_{12}} .
$$

The radius $r_{12}$ is the separation of the center of masses of the two clusters, $M_{1}\left(r_{12}\right)$ is the mass interior to the subcluster at radius $r_{12}, M_{2}$ is the mass of the merging subcluster, and $m_{r}=\left(M_{1} M_{2}\right) /\left(M_{1}+M_{2}\right)$ is the reduced mass. The gravitational potential $\Phi\left(M_{1}, r_{12}\right)$ is defined by

$$
\Phi(M, r)=-\frac{G M(r)}{r} .
$$

The mass interior is defined as

$$
M(r)=4 \pi \xi_{m} \int_{0}^{r} d x x^{2} n_{\mathrm{ICM}}(x),
$$

where $\xi_{m}$ is a normalization constant chosen so that $M_{1}\left(r_{12}\right)$ is equivalent to the total mass of the dominant cluster at its maximum radius $R_{1}$. Obviously, the potential satisfies the requirement $\Phi(M, r \rightarrow \infty)=0$. Initial conditions $(t=0)$ are found by a conservation of energy argument where the initial radius and infall velocity are given by

$$
\begin{gathered}
r_{12}(t=0)=R_{1}+R_{2}, \\
v_{0}(t=0)=\sqrt{\frac{2}{m_{r}}\left[\mathscr{E}-M_{2} \Phi\left(M_{1}, r_{12}\right)\right],}
\end{gathered}
$$

where $R_{2}$ is the maximum radius of cluster $M_{2}$.

In order to test the accuracy of this approach to the dynamics of cluster mergers, we compare the semianalytic values of $r_{12}$ and $v_{0}$ with the results of an $N$-body simulation of a two-cluster merger model (Berrington 2000). For this comparison, we assume that the two clusters have equal masses. This is the worst case, because when one cluster is smaller and less massive than the other, it is better described as a point source. In the $N$-body simulation, each cluster is approximated by $N=80,000$ equal-mass particles, with $50 \%$ of the particles assigned to galaxies in each cluster and the remaining $50 \%$ assigned to a nonluminous dark matter that follows a King-model distribution with $W_{0}=6.25$ (King 1966). The kinetic energy of the particles in each cluster is assumed to be in virial equilibrium with respect to the total gravitational potential energy prior to the interaction.
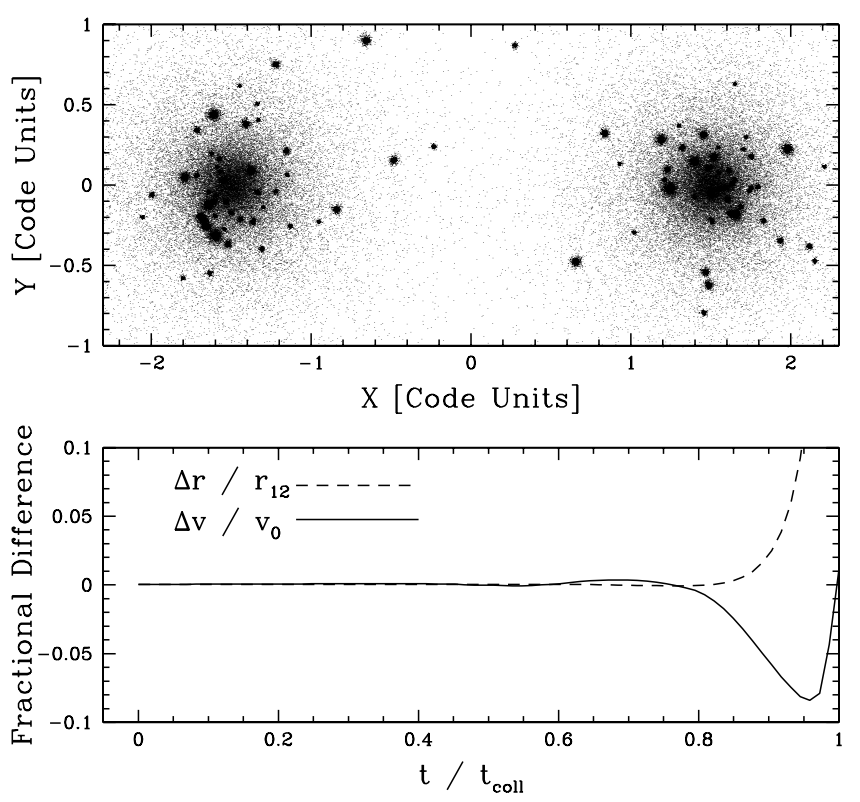

FIG. 1.-Comparison between the semianalytic model used in subsequent calculations and results of an $N$-body simulation. The initial configuration of an $N$-body simulation of a cluster merger between two equal-mass clusters is shown in the top panel. The relative differences $\Delta r$ and $\Delta v$ between the semianalytic and numerical calculations of the centerof-mass distance and the infall velocity of the two clusters are shown in the bottom panel.

Each cluster consists of 50 galaxies with masses that are selected from a Schechter luminosity function with a powerlaw index of -1.25 . The top panel of Figure 1 shows the initial configuration of the merger event, and the bottom panel gives the relative difference between the centers of mass calculated in the $\mathrm{N}$-body simulation and semianalytically.

The calculation ends at the collision time $t_{\text {coll }}$, which is defined as the time when the centers of mass of the two clusters coincide. As can be seen, the semianalytic value of $v_{0}$ is accurate to within $10 \%$ at times preceding $t_{\text {coll }}$. After $t_{\text {coll }}$, the forward shock velocity, $v_{1}$, can no longer be approximated by equation (7) because the shock decouples from the gravitational infall of the merging cluster and is assumed to be constant as the shock propagates outward from the cluster center.

In this paper, we model the cluster merger shocks rather than the accretion shocks. An infalling cluster is assumed to be a virialized, quasi-spherical distribution of galaxies, ICM, and dark matter. A cluster merger shock forms when the cluster merger velocity exceeds the sound speed of the ICM. In contrast, an accretion shock is the result of a spherically symmetric infall of matter from the surrounding volume onto a cluster. In the context of the previous work by Fujita \& Sarazin (2001), our definition of merger events includes not only their definition of a cluster merger event $\left(M_{2}>0.6 M_{1}\right)$ but also their definition of semimerger events $\left(0.6 M_{1}>M_{2}>0.1 M_{1}\right)$. In their calculations, accretion events can account for only $\approx 10 \%$ of the nonthermal photon luminosity. Although each cluster of galaxies is at the center of an accretion flow, we do not consider nonthermal particle production from accretion shocks because their nonthermal photon production is negligible in comparison to cluster merger events. Note that supersonic cluster merger shocks are less likely to form at high redshift for two 
clusters of galaxies with given masses. This is because the relative velocities of the merging clusters will be associated with orbits with smaller separations at earlier times and will therefore have smaller relative velocities. In reality, the mean cluster masses are smaller at early times. These lower mass clusters have a lower virial temperature. If this is considered in the calculation of the strengths of the shocks seen in cluster merger events, it is possible that stronger shocks may be seen at higher redshifts.

\subsubsection{The Cluster Environment}

Shock formation and nonthermal particle production depend on the properties of ICM, which are largely determined by the mass of the cluster and cosmological epoch. Below we describe the equations used to describe the properties of the cluster environment.

X-ray observations of galaxy clusters show that these clusters are permeated by a hot ICM of temperatures in the range of 5-12 keV. At these temperatures the gas is well described by an ideal gas. The sound speed of an ideal gas in terms of the gas temperature $k T_{\mathrm{X}}$ is simply

$$
c_{s}\left(T_{\mathrm{X}}\right)=\sqrt{\frac{\Gamma k T_{\mathrm{X}}}{\mu m_{p}}}=1265 \mu^{-1 / 2}\left(\frac{k T_{\mathrm{X}}}{10 \mathrm{keV}}\right)^{1 / 2} \mathrm{~km} \mathrm{~s}^{-1},
$$

where $\Gamma$ represents the ratio of specific heats, which we take to be equal to $5 / 3, \mu$ is the mean atomic mass, and $m_{p}$ is the proton mass.

The temperature of the ICM depends on both the mass of the cluster and redshift $z$. In order to calculate the cluster $\mathrm{X}$-ray luminosity, which gives the energy of $\mathrm{X}$ rays that can be Compton scattered, we first calculate the expected temperature of the ICM gas. We use the cluster $M-T$ relationship from Bryan \& Norman (1998), given by

$$
k T_{\mathrm{X}}(z)=1.39 f_{T_{\mathrm{X}}}\left(\frac{M_{1}}{10^{15} M_{\odot}}\right)^{2 / 3}\left[\Delta_{c} h^{2} E^{2}(z)\right]^{1 / 3} \mathrm{keV},
$$

where $M_{1}$ is the mass of the cluster, $h$ is the parameter defined by $H=100 h \mathrm{~km} \mathrm{~s}^{-1} \mathrm{Mpc}^{-1}$, the function $E^{2}(z)=$ $\Omega_{0}(1+z)^{3}+\Omega_{R}(1+z)^{2}+\Omega_{\Lambda}$, and $f_{T_{\mathrm{X}}}$ is a normalization constant taken to be 0.8 . We have made use of the following standard definitions:

$$
\Omega_{0}=\frac{8 \pi G \rho_{c}}{3 H_{0}^{2}}, \quad \Omega_{R}=\left(H_{0} R\right)^{-2}, \quad \Omega_{\Lambda}=\frac{\Lambda}{3 H_{0}^{2}},
$$

where $H_{0}$ is the current measured Hubble constant, $\rho_{c}$ is the critical density required to close the universe, $\Lambda$ is the cosmological constant, and $R$ is the current curvature radius of the universe. We force the constraint $\Omega_{0}+\Omega_{R}+\Omega_{\Lambda}=1$ as mandated by an inflationary cosmology. For all models in this paper, we set $\Omega_{R}=0$ for a flat universe. The function $\Delta_{c}$ is the critical density factor and marks the point at which a region of overdensity makes the transition from linear density growth behavior to a nonlinear density growth regime. This marks the birth of a cluster or the beginning of the initial collapse of a density perturbation out of the Hubble flow. Bryan \& Norman (1998) calculate it to be

$$
\Delta_{c}= \begin{cases}18 \pi^{2}, & \Omega_{0}=1, \\ 18 \pi^{2}+82 x-39 x^{2}, & \Omega_{R}=0, \\ 18 \pi^{2}+60 x-32 x^{2}, & \Omega_{\Lambda}=0\end{cases}
$$

where $x=\Omega(z)-1$, and $\Omega(z)=\Omega_{0}(1+z)^{3} / E^{2}(z)$. These relations are accurate to $1 \%$ in the range $\Omega(z)=0.1-1.0$.

A number of radiation fields provide seed photons for the Compton scattering by the nonthermal electrons present in the cluster. For the X-ray radiation field, we calculate the X-ray luminosity of a cluster of mass $M$ by the observed relation (Arnaud \& Evrard 1999)

$$
L_{\mathrm{X}}\left(T_{\mathrm{X}}\right)=2.88 h^{-2} \times 10^{44}\left[\frac{k T_{\mathrm{X}}(z)}{6 \mathrm{keV}}\right]^{2.88} \operatorname{ergs~s}^{-1},
$$

where $k T_{\mathrm{X}}$ is defined by equation (9). Assuming the X-ray luminosity is uniformly distributed within a spherical cluster of radius $R$ and an escape time of $\sim R / c$, the mean energy density of the X-ray photons is

$$
\begin{aligned}
U_{\mathrm{X}}\left(T_{\mathrm{X}}\right) \approx & 1.5 h^{2} \times 10^{-10}\left(\frac{R_{1}}{1 \mathrm{Mpc}}\right)^{-2} \\
& \times\left[\frac{k T_{\mathrm{X}}(z)}{6 \mathrm{keV}}\right]^{2.88} \mathrm{MeV} \mathrm{cm}^{-3} .
\end{aligned}
$$

The thermal X-ray bremsstrahlung is approximated by a blackbody of temperature $T_{\mathrm{X}}$ in our calculations of Compton scattering. This is not a crucial approximation, because the X-ray energy density is $\sim 2-3$ orders of magnitude less than the $\mathrm{CMB}$ energy density, and the importance of Compton-scattered X-ray photons is furthermore reduced because of Klein-Nishina effects.

A second component that contributes to the mean photon energy density of the ICM is the stellar photon field. We assume that the galaxy luminosity function is well approximated by the Schechter luminosity distribution:

$$
n_{\text {gal }}(L)=\frac{\xi_{*}}{L^{*}}\left(\frac{L}{L^{*}}\right)^{-\alpha} \exp \left(-\frac{L}{L^{*}}\right),
$$

where $n_{\text {gal }}(L) d L$ is the differential luminosity function and is defined as the number of galaxies within the luminosity range $L$ and $L+d L$. The parameter $L^{*}$ is the "characteristic luminosity" of a galaxy that marks the transition between the faint-end power law of slope $-\alpha$ and the highend exponential cutoff. The constant $\xi_{*}$ normalizes the Schechter function to $N_{\text {gal }}$ when integrated over all luminosities. We can use equation (14) to calculate the total stellar luminosity of

$$
L_{\text {star }}=\int_{0}^{\infty} d L \operatorname{Ln}_{\mathrm{gal}}(L)=\xi_{*} \Gamma(2-\alpha) L^{*},
$$

where $\Gamma(2-\alpha)$ is the gamma function. Typical values for $\xi_{*}, L^{*}$, and $\alpha$ are given, e.g., in Paolillo et al. (2001), and our adopted values are $\alpha=1.1$, a value of $L^{*} \cong 7 \times 10^{43}$ ergs $\mathrm{s}^{-1}$ corresponding to an absolute magnitude $M^{*}=-20.5$ in $B_{J}$ (Colless 1989), and $\xi_{*}=100$.

Using the same approach as for the X-ray photon field, the estimated mean stellar radiation field becomes

$$
U_{\text {star }} \approx 1.4 h^{2} \times 10^{-9}\left(\frac{R_{1}}{1 \mathrm{Mpc}}\right)^{-2} \mathrm{MeV} \mathrm{cm}^{-3} .
$$

The spectral distribution of elliptical galaxies is well approximated by a K3 III star (Pierce \& Berrington 2003). Since higher density regions in rich galaxy clusters are dominated by elliptical galaxies, we assume that the stellar radiation energy distribution can be approximated by a 
blackbody spectrum resembling that of a K3 III star at temperature $T_{\text {star }} \approx 4000 \mathrm{~K}$.

The energy density of the $\mathrm{CMB}$ is

$$
U_{\mathrm{CMB}}=2.5 \times 10^{-7}(1+z)^{4} \mathrm{MeV} \mathrm{cm}^{-3} .
$$

The $\mathrm{CMB}$ is the dominant contributor to total cluster photon energy density by approximately 3 orders of magnitude. The total cluster radiation field is simply the sum of equations (13), (16), and (17).

\subsubsection{Cluster Redshift}

The cluster environment described in $\S 2.1 .2$ depends on the redshift of the cluster. The redshift evolution of the cluster depends on the chosen cosmological model. We have chosen to uniquely specify the cosmological model by the following parameters: the critical mass fraction $\Omega_{0}$, the curvature $\Omega_{R}$, the dark energy $\Omega_{\Lambda}$, and the Hubble constant $H_{0}$. For this paper we consider the model $\left(\Omega_{0}, \Omega_{R}, \Omega_{\Lambda}\right)=$ $(0.3,0.0,0.7)$.

The observed redshift of a cluster is determined from the time since the big bang by the cosmological equation (Peebles 1993)

$$
\frac{d z}{d t}=-H_{0}(1+z) E(z),
$$

where $E^{2}(z)$ is the dimensionless parameter defined following equation (9). Typical propagation times for a shock front to traverse a cluster are $\sim 10^{9} \mathrm{yr}$. This is a significant time span in that the evolution of the cluster environment due to cosmic expansion must be considered. Cluster merger events can be initiated at any time throughout age of the universe, and evolving the cluster environment is necessary to accurately calculate the nonthermal photon and particle spectra.

\subsection{Nonthermal Particle Production}

Henceforth, we denote particle kinetic energies by $K_{e, p}=m_{e, p}\left(\gamma_{e, p}-1\right) c^{2}$, total energies by $E_{e, p}$, and dimensionless total energies by $\epsilon_{i}$, with the subscripts $e$ and $p$ referring to electrons and protons, respectively. First-order Fermi acceleration at a cluster merger shock is approximated by injecting power-law momentum spectra for the electron and protons. The total particle injection function in terms of kinetic energy is given by

$$
\begin{aligned}
Q_{e, p}\left(K_{e, p}, t\right)= & Q_{e, p}^{0}\left[K_{e, p}\left(K_{e, p}+2 m_{e, p} c^{2}\right)\right]^{-[s(t)+1] / 2} \\
& \times\left(K_{e, p}+m_{e, p} c^{2}\right) \exp \left[-\frac{K_{e, p}}{K_{\max }(t)}\right],
\end{aligned}
$$

where $s(t)$ is the injection index and $K_{\max }$ is the maximum particle energy determined by three conditions: the available time to accelerate to a given energy since the beginning of the merger event, the requirement that the particle Larmor radius is smaller than the size scale of the system, and the condition that the energy-gain rate through firstorder Fermi acceleration is larger than the energy-loss rate due to synchrotron and Compton processes. The duration of particle injection is determined by the dynamics of the system, and the shock infall velocity is determined by equation (4). The shocks terminate at the time $t_{\mathrm{acc}, 1}$ for the forward shock and $t_{\text {acc, }, 2}$ for the reverse shock when the shock propagates to the outer part of the cluster. The time $t_{\text {acc }}$ represents the greater value of two acceleration times $t_{\mathrm{acc}, 1}$ and $t_{\mathrm{acc}, 2}$. The constant $Q_{e, p}^{0}$ normalizes the injected particle spectrum over the volume swept out by the shock front and is determined by

$$
\int_{K_{\min }}^{K_{\max }} d K_{e, p} K_{e, p} Q_{e, p}\left(K_{e, p}, t\right)=\frac{\eta_{e, p}}{2} A \eta_{\mathrm{He}}^{e} m_{p} v_{1}(t)^{3}\left\langle n_{\mathrm{ICM}}(t)\right\rangle,
$$

where $A$ is the area of the shock front, $\eta_{\mathrm{He}}^{e} \cong 1.2$ is an enhancement factor due to the presence of ions heavier than hydrogen, and $\left\langle n_{\mathrm{ICM}}(t)\right\rangle$ is the number density of the gas averaged over the area of the shock front at time $t$. We assume a constant efficiency factor $\eta_{e, p}=5 \%$ for both electrons and protons, although $\eta_{e, p}$ would depend on Mach number through the uncertain wave generation processes at the shock front. ${ }^{1}$ The particle injection index, $s(t)$, is determined by the shock properties described in $\S 2.2 .1$. Typical values for the particle injection index are $s \approx 2.5$.

The temporally evolving particle spectrum is determined by solving the Fokker-Planck equation in energy space for a spatially homogeneous ICM, given by

$$
\begin{aligned}
\frac{\partial N(K, t)}{\partial t}= & \frac{1}{2} \frac{\partial^{2}}{\partial K^{2}}[D(K, t) N(K, t)] \\
& -\frac{\partial}{\partial K}\left\{\left[\dot{K}_{\mathrm{tot}}(K, t)-A_{\mathrm{tot}}(K, t)\right] N(K, t)\right\} \\
& -\sum_{i=p p, p \gamma, d} \frac{N(K, t)}{\tau_{i}(K, t)}+Q(K, t) .
\end{aligned}
$$

The quantity $\dot{K}_{\text {tot }}$ represents the total kinetic-energy loss rate, and $A_{\text {tot }}$ represents the total energy gain rate. Electrons experience energy losses from synchrotron, bremsstrahlung, Compton, and Coulomb processes. The total continuous energy-loss rate for protons is due to the effects of Coulomb processes. In addition, protons experience catastrophic energy losses due to proton-proton collisions $(i=p p)$, proton- $\gamma$ collisions $(i=p \gamma)$, and diffusive escape $(i=d)$ on the timescale $\tau_{i}\left(K_{p}, t\right)$. The spectra of the secondary electrons are calculated from the pion-decay products (see Appendix A.3), and are subject to the same energy losses as the primary electrons. The calculation of the energy-loss rates accounted for by the quantity $\dot{K}_{\text {tot }}$ for both electrons and protons is presented in detail in Appendix A. The nonthermal energy spectra resulting from the temporal evolution of equation (21) will produce a nonthermal photon spectra. The method used to calculate the synchrotron, bremsstrahlung, Compton, and $\pi_{0} \gamma$-ray radiation components to the photon energy spectra is presented in detail in Appendix B.

The integration of the partial differential equation (21) poses a significant computational problem when considering the ranges of energies modeled in this paper. The problem stems from the diffusion that occurs at low energies and the large energy losses that occur at high energies. We found that equation (21), when solved with a Crank-Nicholson implicit finite differencing method, is unstable to an oscillation instability at the high and low energies because of the large values that the convection derivative takes at both

\footnotetext{
${ }^{1}$ A quantitative prescription of the shock efficiency as a function of the injection index is given by Miniati et al. (2001); see also Keshet et al. (2003) on this issue.
} 
energy extremes. To solve equation (21), we settled on an implicit, upwind finite differencing method. This has the advantage of solving the oscillation instability problem, although with the disadvantage of a decrease in numerical accuracy for a given step size.

\subsubsection{Particle Source Function}

Nonthermal particle distributions are injected into the ICM according to the function given by equation (19). The normalization constant $Q_{e, p}^{0}$ is calculated as described in $\S 2.2$. The particle distribution functions have spectral index $s(t)$ and exponential cutoffs at kinetic energy $K_{\max }$, which are determined by shock acceleration theory as described below.

Nonthermal particles gain energy through first-order Fermi shock acceleration at the rate

$$
\dot{K}_{\mathrm{sh}}(t)=100 \frac{B v_{8}^{2}(t)}{f r_{J}} \mathrm{MeV} \mathrm{s}^{-1}
$$

(Sturner et al. 1997; Reynolds 1996), where $v_{8}(t)=v_{1}(t) /$ $\left(10^{8} \mathrm{~cm} \mathrm{~s}^{-1}\right)$ is the shock velocity in units of $10^{3} \mathrm{~km} \mathrm{~s}^{-1}, f$ is a factor giving the mean free path of a particle in units of its gyroradius, and the quantity $r_{J}$ accounts for the orientation of the shock front relative to the magnetic field. In our calculations, we set $f=10$ and $r_{J}=1$.

The first constraint that we impose on maximum particle energy is the available time to accelerate particles to a given energy. This constraint is determined through the expression

$$
K_{\max , 1}=\int_{0}^{t} d t \dot{K}_{\mathrm{sh}}(t),
$$

where $v_{1}(t)$ is found from equation (4) and $B$ is assumed to be constant throughout the cluster volume. This constraint is the most important limitation on proton energy for most of the lifetime of the shock front.

Competition with energy losses sets a second constraint on maximum particle energy. By equating the electron energy gain rate, equation (22), with the sum of the synchrotron and Compton energy losses that are the dominant electron energy-loss processes at high energies, we obtain a second energy constraint for electrons, given by

$$
K_{\max , 2}=2.8 \times 10^{7} \frac{v_{8}(t)}{\sqrt{f r_{J}}}\left[\frac{B_{-7}}{(1+z)^{4}+10^{-3} B_{-7}^{2}}\right]^{1 / 2} \mathrm{MeV},
$$

where $B_{-7}$ is the magnetic field in units of $10^{-7} \mathrm{G}$. Considering synchrotron losses only, the corresponding maximum energy for protons is

$$
K_{\max , 2(\text { syn })} \cong 2 \times 10^{15} \frac{v_{8}(t)}{\sqrt{B_{-7} f r_{J}}} \mathrm{MeV} .
$$

For the parameters used in our calculations, proton energy losses due to Compton and synchrotron processes are smaller than photomeson and photopair losses with the CMB. These latter processes could in principle constrain the maximum proton energy (Berezinskii \& Grigor'eva 1988) but are found to be negligible for the parameters used in the calculations. This is because the timescale to accelerate protons to ultrahigh energies $\left(\gtrsim 10^{19} \mathrm{eV}\right)$ requires $\approx 3 \times 10^{11}\left[f r_{J} / B_{-7} v_{8}^{2}(t)\right]\left(K_{p} / 10^{20} \mathrm{eV}\right)$ yr, which is longer than the Hubble time $t_{H}$ and much longer than the timescale over which the merger shocks persist in the merging clusters $\left(\approx 0.1 t_{H}\right)$. Although photomeson production does not pose a significant limitation to particle acceleration, it is still treated as a catastrophic loss in the calculation.

In order for particle acceleration to occur, the Larmor radius of a particle must be smaller than the size scale of the system; otherwise, the particle escapes the system before any significant energy gain can occur. The third constraint imposed on maximum particle energy is therefore obtained by requiring that the particle Larmor radius be less than the size scale of the shock front. This constraint becomes, for both protons and electrons,

$$
K_{\max , 3}=\frac{e B \lambda}{f} \cong \frac{10^{13}}{f} B_{-7}\left(\frac{\lambda}{\mathrm{Mpc}}\right) \mathrm{MeV},
$$

where $\lambda=2 R_{2}$ and $e$ is the electron charge. In all of our calculations, the limitations imposed by $K_{\max , 1}$ and $K_{\max , 2}$ are more restrictive for the electrons. However, for protons, $K_{\max , 3}$ is the strongest limitation for the highest energy protons at the later stages of the shock evolution. The maximum energy $K_{\max }(t)$ in equation (19) is the minimum of the maximum energies calculated in equations (23)-(26). Note that the size-scale limitation on maximum particle energy assumes that the coherence length of the magnetic field is larger than the size scale of the shock.

The particle index $s(t)$ is determined from the collision and sound speeds of the system. The collision speed $v_{0}(t)$ is obtained by solving equation (4) with the initial radius of the interaction given by the sum of the assumed radii of the clusters and the initial collision velocity given by equation (7). We assume that the subcluster is a point mass and the dominant cluster is described by the isothermal beta model density profile given in equation (3).

The shock compression ratio is calculated from the equation

$$
\mathscr{C}(t)=\frac{\Gamma+1}{\Gamma-1+2 \mathscr{M}^{-2}(t)},
$$

where $\mathscr{M}(t)=v(t) / c_{s}$ is the Mach number of the cluster merger shock speed, as computed from equations (4), (30), and (31). The ratio of specific heats is represented by $\Gamma=5 / 3$, appropriate to a nonrelativistic monoatomic ideal gas. In Figure 2, the time evolution of the compression ratio of a shock front in a merging cluster with masses $M=10^{15}$ $M_{\odot}$ and $m=10^{14} M_{\odot}$ is graphed. The radii of the two systems are assumed to be 1.5 and $0.75 \mathrm{Mpc}$, and the onset of the merger is at a redshift of $z_{i}=0.1$. From the shock compression ratio, we calculate the injected particle index $s$ from

$$
s(t)=\frac{\mathscr{C}(t)+2}{\mathscr{C}(t)-1} .
$$

The calculated shock speeds of cluster merger shocks range from $\sim 1.5$ to 3 times the ICM sound speed, with power-law indexes ranging from $\sim 5$ to $\sim 2.3$. Figure 2 also shows the evolution of the typical power-law index as a function of time.

In the merger event between two clusters of galaxies, as described in $\S 2.1$, two shock fronts develop. The shock front propagating into the dominant cluster, which we call the forward shock, has Mach number $\mathscr{M}_{1}(t)$. Similarly, the shock front propagating into the merging cluster has Mach number $\mathscr{M}_{2}(t)$. Writing down the compression ratio for the 


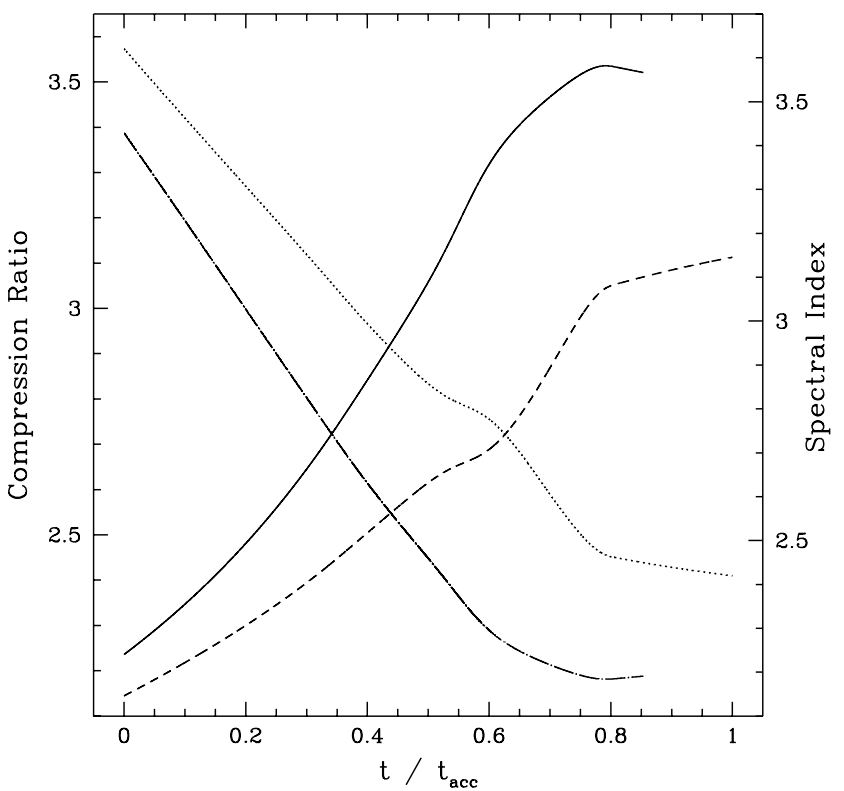

FIG. 2.-Compression ratio of the forward (dashed curve) and reverse (solid curve) shocks as calculated by the method described in $\S 2.2 .1$. The particle injection spectral index for the forward (dotted curve) and reverse (dot-dashed curve) shocks is associated with the compression ratio and is calculated for a merger system consisting of a dominant cluster of mass $10^{15} M_{\odot}$ and a poor subcluster of mass $10^{14} M_{\odot}$. Initial onset of the shock begins at a radius of $1.5 \mathrm{Mpc}$ from the cluster center with an initial redshift of $z_{i}=0.1$. Particle acceleration occurs over a period $t_{\mathrm{acc}}=1.1 \mathrm{Gyr}$ for these parameters, after which injection stops. Acceleration at the reverse shock ends after the reverse shock passes through the merging cluster.

forward $\left[\mathscr{C}_{1}(t)\right]$ and reverse $\left[\mathscr{C}_{2}(t)\right]$ shocks from equation (27), we get

$$
\begin{aligned}
& \mathscr{C}_{1}(t)=\frac{u_{1}(t)}{v}=\frac{4}{3\left[1-\mathscr{M}_{1}^{-2}(t)\right]}, \\
& \mathscr{C}_{2}(t)=\frac{u_{2}(t)}{\bar{v}}=\frac{4}{3\left[1-\mathscr{M}_{2}^{-2}(t)\right]},
\end{aligned}
$$

where $u_{1}(t)$ and $u_{2}(t)$ are the velocities of the forward and reverse shocks, respectively. In addition, $v$ and $\bar{v}$ are the velocities of the shocked fluid for the forward and reverse shocks, respectively. Noting that $\bar{v}=v_{0}(t)-v$, where $v_{0}$ is the infall velocity of the merging cluster, and solving for the Mach number, we get

$$
\begin{gathered}
\mathscr{M}_{1}(t)=\frac{2}{3} \frac{v}{c_{1}}\left(1+\sqrt{1+\frac{9}{4} \frac{c_{1}^{2}}{v^{2}}}\right), \\
\mathscr{M}_{2}(t)=\frac{2}{3} \frac{v_{0}(t)-v}{c_{2}}\left(1+\sqrt{1+\frac{9}{4} \frac{c_{2}^{2}}{\left[v_{0}(t)-v\right]^{2}}}\right),
\end{gathered}
$$

where $c_{1}$ is the sound speed in the dominant cluster, $c_{2}$ is the sound speed in the merging cluster, and the Mach number is defined to be $\mathscr{M}_{1,2}=u_{1,2} / c_{1,2}$. The values of $c_{1}$ and $c_{2}$ are calculated from equation (8) with temperatures given by equation (9) for clusters of mass $M_{1}$ and $M_{2}$. The value of $v$ is calculated by solving

$$
\frac{\mu_{1}}{\mu_{2}} \frac{n_{1}(t)}{n_{2}(t)}=\frac{1+3 \mathscr{M}_{1}^{-2}(t)}{1+3 \mathscr{M}_{2}^{-2}(t)}\left[\frac{v_{0}(t)-v}{v}\right]^{2},
$$

where $n_{1}(t)$ and $n_{2}(t)$ are the ICM number densities in the dominant cluster and merging cluster at the positions of the forward and reverse shocks, respectively, and are calculated according to equation (3). We take the mean mass per particle $\mu_{1}=\mu_{2}=0.6 m_{p}$ in the two clusters. Equation (31) was derived from the shock jump conditions by equating the energy densities of the shocked fluids at the contact discontinuity. The compression ratios are then calculated from equation (27). These values are plotted in Figure 2.

\section{RESULTS}

We present results of the numerical model described in $\S 2$ to simulate the temporal evolution of nonthermal particles accelerated by shocks formed in merging clusters of galaxies. From these particle distributions, we are able to investigate the expected nonthermal photon spectra for energies ranging from $\sim 10^{-9}$ to $\sim 10^{19} \mathrm{eV}$ that cover astrophysically interesting frequencies. At photon energies $\lesssim 4 \times 10^{-8} \mathrm{eV}$ photons $(\approx 10 \mathrm{MHz})$, ground-based radio observations are subject to the ionospheric absorption cutoff. At photon energies $\gtrsim 10^{12} \mathrm{eV}$ (depending precisely on source redshift), the universe becomes opaque to $\gamma$-rays due to pair production attenuation on the diffuse infrared radiation fields. The electron-positron pairs produced by the $\sim 10^{14}$ to $\sim 10^{19} \mathrm{eV}$ photons provide a secondary source of synchrotron and Compton-scattered radiation on the Mpc galaxy-cluster scale, but this cascade radiation is found to provide only a minor nonthermal synchrotron component to the nonthermal UV/soft X-ray emission and is not included. To calculate detectability of clusters with ground-based Cerenkov telescopes, $\gamma \gamma$ absorption of the high-energy $\gamma$ rays must, however, be considered.

The energy-loss timescales of nonthermal electrons for various parameters assumed in cluster environments are shown in Figure 3. At low energies, Coulomb losses dominate. A maximum in the energy-loss timescale is found in the energy range from $\approx 1$ to several hundred $\mathrm{MeV}$. At higher energies, Compton and synchrotron losses dominate. At the highest energies, the Compton loss rate declines because of the onset of the Klein-Nishina reduction in the Compton cross section, and synchrotron losses become the dominant loss mechanism (see also Sarazin 1999). The electron loss-rate from double Compton scattering is always much smaller than synchrotron losses in our study and can be safely neglected (see $\S$ A.1).

Figure 4 shows the nonthermal particle energy spectra at different times during the merger of a dominant cluster of mass $M_{1}=10^{15} M_{\odot}$ and radius $R_{1}=1.5 \mathrm{Mpc}$ with a subcluster of mass $M_{2}=10^{14} M_{\odot}$ and a radius $R_{2}=0.75 \mathrm{Mpc}$, respectively. The quantities $M_{1}, M_{2}, R_{1}$, and $R_{2}$ are defined in $\S 2$.1.1. The assumed core radius $r_{c}$ for the dominant cluster is $250 \mathrm{kpc}$. The ICM density of the dominant cluster is assumed to follow equation (3) with a central number density $n_{0}=10^{-3} \mathrm{~cm}^{-3}$ and $\beta=0.75$. These parameters are consistent with the $L_{X}-T_{X}$ relation (eq. [12]). A uniform magnetic field of strength $1 \mu \mathrm{G}$ was assumed throughout the cluster. The galaxy cluster environment and its evolution is calculated using the cosmological parameters $\left(\Omega_{0}, \Omega_{R}, \Omega_{\Lambda}\right)=(0.3,0.0,0.7)$. All models assume a Hubble constant $H_{0}=70 \mathrm{~km} \mathrm{~s}^{-1} \mathrm{Mpc}^{-1}$. We also assume a hydrogen-to-helium number ratio of 10 .

Irrespective of the specific parameters of the simulation, we see that at energies $\lesssim 1 \mathrm{MeV}$, the electron and proton 

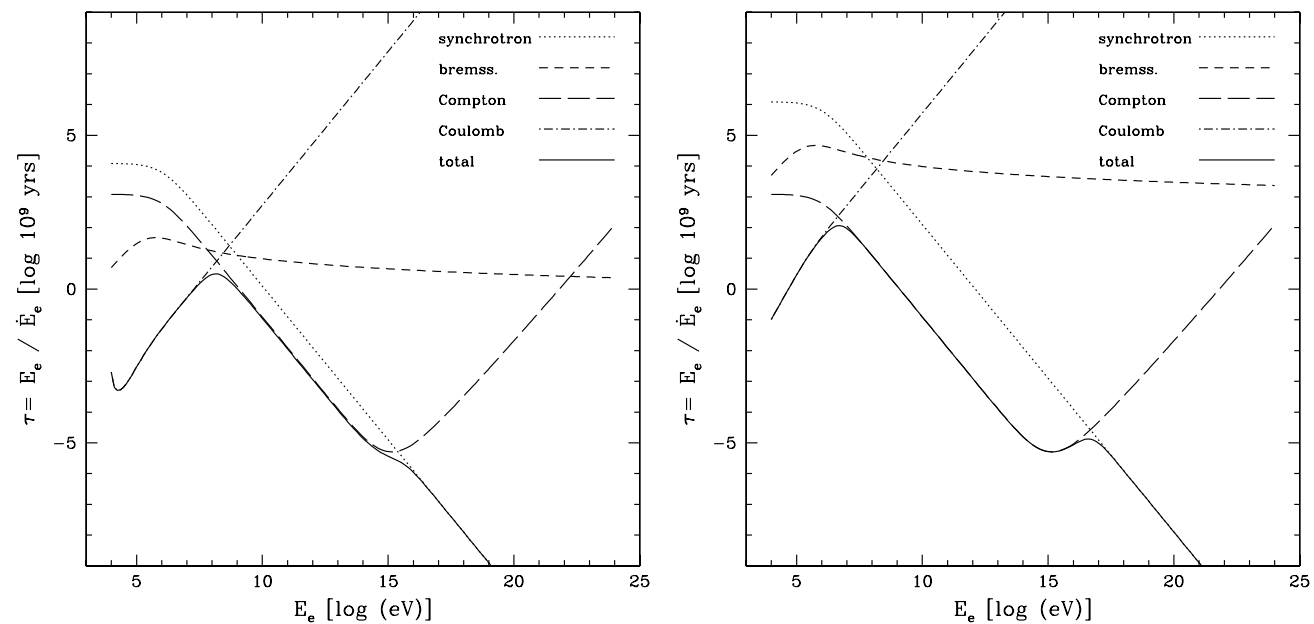

Fig. 3.- Instantaneous energy loss timescale for electrons in a merging cluster scenario at redshift $z=0.0$. In the left panel, the proton density $n=10^{-3} \mathrm{~cm}^{-3}$, and $B=1 \mu \mathrm{G}$. Only the dominant $\mathrm{CMB}$ radiation field is considered. In the right panel, $n=10^{-6} \mathrm{~cm}^{-3}$, and $B=0.1 \mu \mathrm{G}$.

spectra display low-energy cutoffs due to Coulomb diffusion and energy losses (the thermal particle distributions are not shown). Electrons with energies greater than $\sim 1 \mathrm{GeV}$ lose a significant fraction of their energy on Gyr timescales. The dominant energy-loss mechanism for these electrons is Compton scattering, as Thomson losses on the CMB dominate synchrotron losses when $B \ll 3(1+z)^{2} \mu \mathrm{G}$ (see Fig. 3). The dominant energy-loss mechanism for protons is secondary nuclear production, which operates on a timescale of about $2 t_{H}$ for an ambient density of $10^{-3} \mathrm{~cm}^{-3}$. Consequently, the high-energy cutoff in the proton distribution function results from the available time to accelerate particles and, at the longest times, competition of particle acceleration with catastrophic $p \gamma$ losses. Even though particles are injected with a range of power-law indexes, most of the injection power occurs when the spectral index is hardest, yielding a proton spectrum that can be accurately described by a single power law (compare Fig. 2) with a number index of $\approx-2.5$ for the forward shock and $\approx-2.2$ for the reverse shock. The electron bremsstrahlung and secondary nuclear production assume a target proton density of $10^{-3} \mathrm{~cm}^{-3}$.
Separate radiation components produced by nonthermal particles are shown in Figure 5 for a shock formed in the merger of $10^{14}$ and $10^{15} M_{\odot}$ clusters, with the interaction beginning at $z_{i}=0.3$. The system has been evolved for 0.8 $\operatorname{Gyr}(z=0.22)$ in Figures $5 a$ and $5 b$ and for $3.42 \operatorname{Gyr}(z=0)$ in Figure $5 c$. Figures $5 a$ and $5 b$ correspond to times before particle injection has stopped. The cluster magnetic field strength $B=1.0 \mu \mathrm{G}$ in Figures $5 a$ and $5 c$ and $B=0.1 \mu \mathrm{G}$ in Figure $5 b$. The radiation components of the primary and secondary electrons are shown by the short-dashed and dotted curves, respectively, and the $\pi^{0}$-decay $\gamma$-rays are shown by the dotted curves. The curves are labeled by the various radiation processes, and the total spectral energy distributions are shown by the solid curves. The synchrotron and Compton radiations from primary electrons dominate the emission at times before primary injection stops, although $\pi^{0}$-decay $\gamma$-rays make a dominant contribution above 100 $\mathrm{TeV}$. At late times after particle injection ceases, the cooling primary electrons provide the dominant contribution at the lowest radio frequencies and at optical/UV frequencies, but secondary electrons can make the dominant contribution at
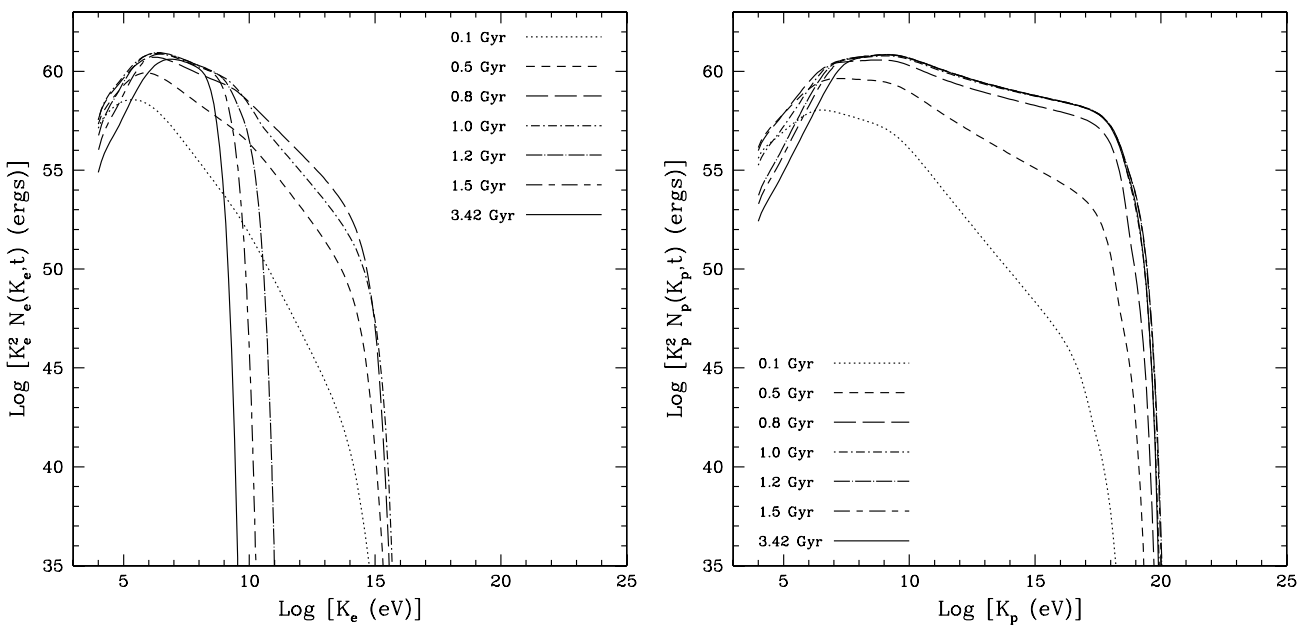

Fig. 4.-Primary electron (left) and proton (right) energy spectra for a shock formed by a merging cluster using a standard parameter set with $M_{1}=10^{15}$ $M_{\odot}, M_{2}=10^{14} M_{\odot}$, and $B=1.0 \mu \mathrm{G}$. The interaction begins at redshift $z_{i}=0.3$ and is evolved to the present time $(z=0.0 ;$ solid curve $)$ with spectra shown at the various times given in the figure. The redshifts corresponding to the time delays of $0.1,0.5,0.8,1.0,1.2,1.5$, and $3.42 \mathrm{Gyr}$ are $z=0.289,0.248,0.218,0.199$, $0.180,0.153$, and 0.0 , respectively. Particle injection ends at $t_{\text {acc }} \cong 1.1 \mathrm{Gyr}$ (see discussion following eq. [19]). 

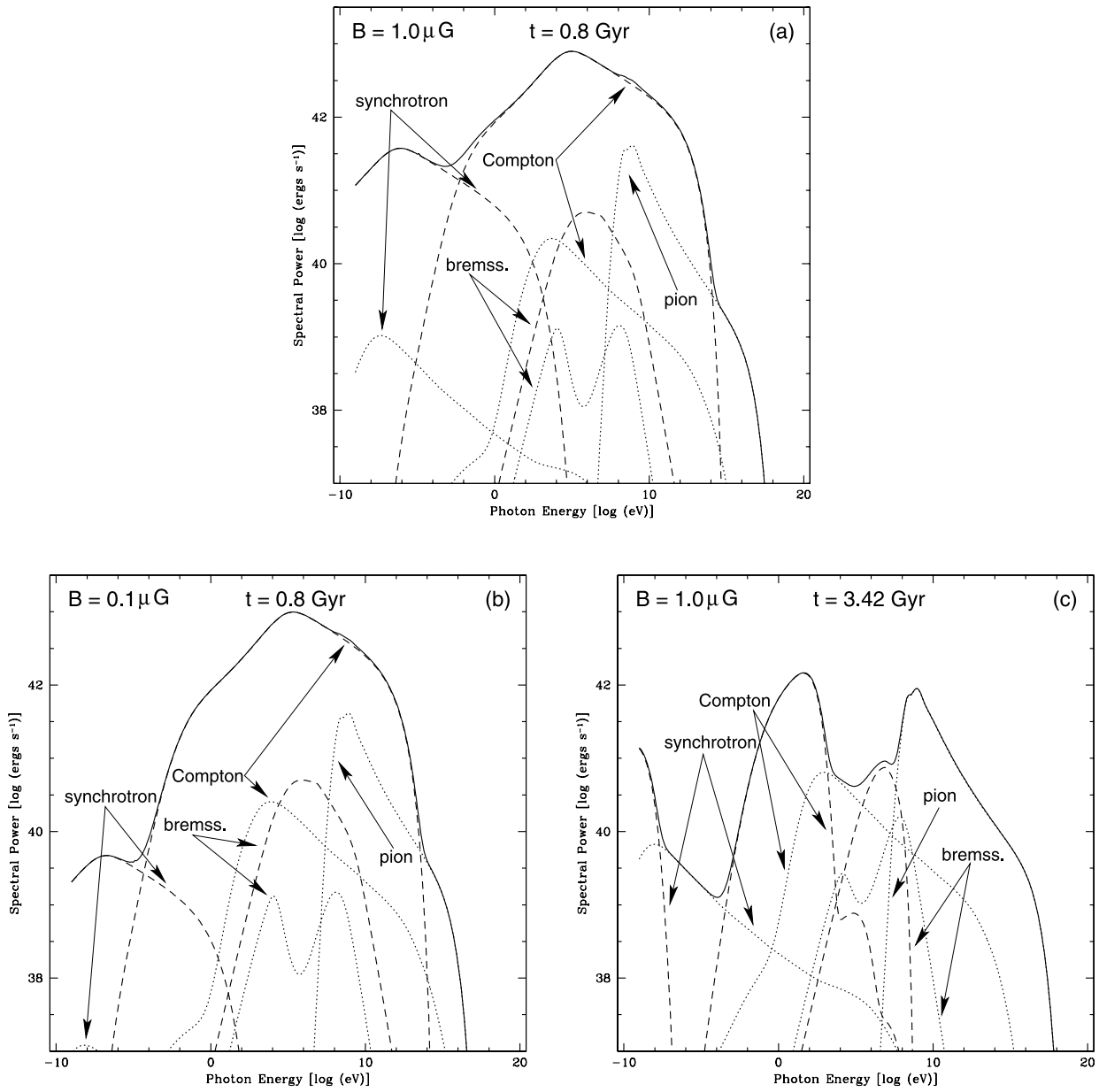

FIG. 5.- Separate radiation components produced by nonthermal particles accelerated by a shock initiated at $z_{i}=0.3$ resulting from the merger between $10^{14}$ and $10^{15} M_{\odot}$ clusters. The separate radiation components are labeled in the figures. Emission from the primary electrons are represented by the dashed curves, and emission from secondary electrons and positrons and $\pi^{0} \gamma$-rays are represented by the dotted curves. The solid curves are the total nonthermal photon spectra. Panels $a$ and $b$ show the nonthermal spectrum at $0.8 \mathrm{Gyr}(z=0.22)$, and panel $c$ at $3.42 \mathrm{Gyr}(z=0.0)$ after the merger shock forms. Magnetic field strengths are $B=1.0 \mu \mathrm{G}$ for panels $a$ and $c$ and $B=0.1 \mu \mathrm{G}$ for panel $b$. Panels $a$ and $b$ correspond to times before the termination of the nonthermal particle injection. Panel $c$ is approximately $2.3 \mathrm{Gyr}$ after particle injection has ceased.

1.4 GHz. At $\gamma$-ray energies, primary bremsstrahlung and secondary $\pi^{0}$-decay $\gamma$-rays make the dominant contribution at late times. Note that structure in some of the emission components is due either to knock-on electrons or to the separate effects of the stellar, X-ray, and CMB Compton energy losses on the electron energy spectra. As can be seen, the intensity of the synchrotron radio emission is very sensitive to the magnetic field strength.

Figure 6 shows the temporal evolution of the total photon spectra radiated by a shock formed in the merger of $10^{14}$ and $10^{15} M_{\odot}$ clusters with $B=1.0 \mu \mathrm{G}$. The photon spectra are shown at various times for shocks that begin at redshifts $z_{i}=0.03,0.05,0.1$, and 0.3 . Each shock is evolved to the present time $(z=0.0)$, and the radiation spectrum at the present time is represented by the solid curve. Typical lifetimes for the shocks are $\approx 1$ Gyr. As a result, only shock fronts with $z_{i} \geq 0.1$ show late-time evolution of the total photon spectra.

At early times well before particle injection ceases $(\lessgtr 0.5$ Gyr), the luminosity increases as the shock front accelerates particles and the particles accumulate. During this epoch, the injection spectrum is very soft, and nonthermal brems- strahlung and $\pi^{0}$-radiation can dominate the production of photons at high energies because of the relatively few highenergy electrons. The peaks in the spectra at $t<0.3 \mathrm{Gyr}$ spectra are, from low to high energies, due to Compton, bremsstrahlung, and $\pi^{0}$-radiation. The enhancement at low frequencies is due to nonthermal synchrotron radiation. As the front ages and the electron spectrum hardens (0.5-1 Gyr), the number of high-energy electrons increases, and the Compton process strongly dominates the nonthermal emission at infrared energies and above.

At times between $t_{\text {coll }}$ and $t_{\text {acc }}$ (between $\approx 0.9$ and $1.1 \mathrm{Gyr}$ in Fig. 6), the shock front propagates to the outer regions of the clusters, and the particle injection rate decreases. The number of high-energy electrons begins to decrease as their previous numbers are no longer replenished. The consequence of this behavior is a softening in the synchrotron and high-energy Compton emission. This is seen clearly in the $z_{i}=0.1$ and $z_{i}=0.3$ panels. As the Compton emission declines, the $\gamma$-rays from the $p+p \rightarrow \pi_{0} \rightarrow 2 \gamma$ process become increasingly evident at times $\gtrsim 1.1 \mathrm{Gyr}$.

The shock front terminates at $t_{\mathrm{acc}}$ after propagating through the cluster. At these late times, particle injection 

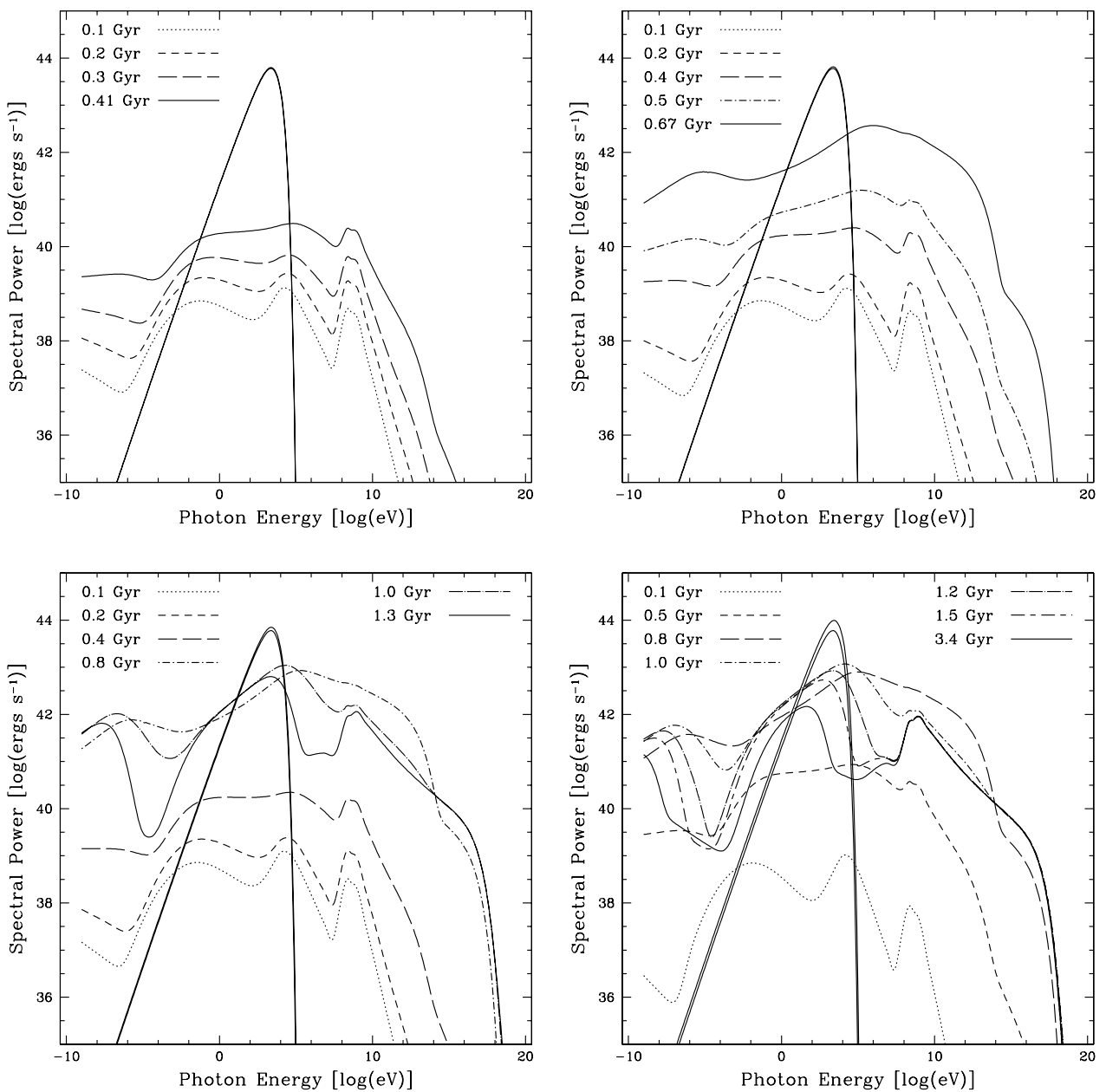

Fig. 6. - Temporal evolution of the total spectral energy distribution for a cluster merger shock that begins at redshift $z_{i}=0.03$ (top left), $z_{i}=0.05$ (top right), $z_{i}=0.1$ (bottom left), or $z_{i}=0.3$ (bottom right). Each shock is evolved to the present (solid curve). Intermediate times are indicated in each panel. In all cases the shock survives for $\sim 1.1$ Gyr. Cluster merger shocks that develop at redshifts $\gtrsim 0.1$ do not persist to the present epoch. The thin solid curve shows the expected thermal bremsstrahlung for a $10^{15} M_{\odot}$ cluster, whose temperature is given by eq. (9). The figures with two thin solid curves represent the thermal bremsstrahlung at $z_{i}$ (lower curve) and $z=0.0$ (higher curve). The integrated luminosity of the thermal bremsstrahlung is $\approx 10^{45} \mathrm{ergs} \mathrm{s}^{-1}$.

ceases, and the remaining population of nonthermal particles evolve according to the energy loss and diffusion processes described in $\S \S \mathrm{A} 1$ and A2, with no freshly accelerated electrons to repopulate the particle distribution. The system quickly becomes devoid of primary electrons, so that the bremsstrahlung and pion emission dominate at HXR energies and above. The most extreme case is shown in the $z_{i}=0.3$ panel of Figure 6 . The spectrum at the present epoch shows three maxima produced by synchrotron radiation at $\mathrm{kHz}$ frequencies, Compton scattering near $100 \mathrm{eV}$, and combined nonthermal bremsstrahlung and $\pi_{0}$-decay emission near $100 \mathrm{MeV}$. The plateau in the radio emission at late times is formed by synchrotron emission from secondary electrons $\left(1.4 \mathrm{GHz}\right.$ corresponds to $\left.6 \times 10^{-6} \mathrm{eV}\right)$.

Also shown in Figure 6 is the thermal bremsstrahlung emission from the ICM using the form for the emission spectrum given by Rybicki \& Lightman (1979). The temperature and the luminosity of the thermal bremsstrahlung is computed from equations in $\S 2.1 .2$. Thermal bremsstrahlung generally dominates the emission at $10 \mathrm{eV}$ to $10 \mathrm{keV}$ energies. It should be noted that the relations for temperature and luminosity of the thermal bremsstrahlung component do not take into consideration the merging process of the system. Computer simulations of expected X-ray temperatures and luminosities of merging clusters indicate an increase in the thermal bremsstrahlung luminosity and temperature (Ricker 1998; Schindler \& Müller 1993). Therefore, the thermal bremsstrahlung curves presented in Figures 6 and 7 very likely underestimate the true X-ray luminosity and temperature of the gas during the merger event. At times $\gg t_{\mathrm{acc}}$, the relation between the initial temperature and luminosity will depend on mass ratio of the merger components, the dark-matter density profile of the clusters, and impact parameter of the merger event (Ricker \& Sarazin 2001).

Figure 7 shows a calculation for the same system as in Figure 6, except for a cluster with a magnetic field of $0.1 \mu \mathrm{G}$. We consider these two values because of the uncertainty regarding the mean magnetic fields in clusters of galaxies. Despite Faraday rotation measurements indicating magnetic fields strengths $B \gtrsim 1.0 \mu \mathrm{G}$ (Clarke, Kronberg, \& Böhringer 2001), a comparison of radio and Compton luminosities suggests field strengths $B \approx 0.1 \mu \mathrm{G}$ (FuscoFemiano et al. 1999). Possible explanations for this discrepancy may arise from an observational biasing of the Faraday rotation measurement of the magnetic fields- 

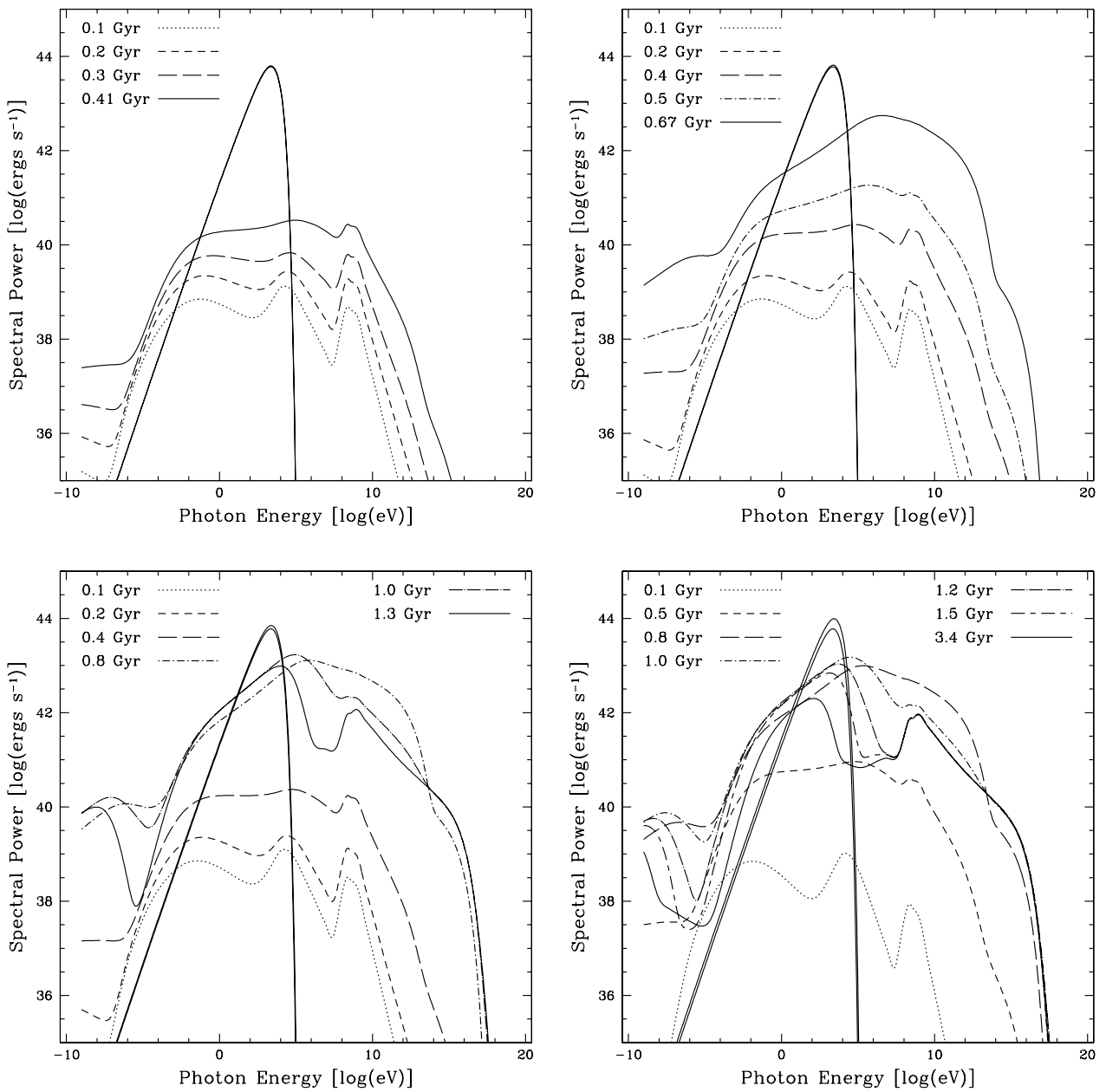

FIG. 7.-Same as Fig. 6, except with an assumed cluster magnetic field of $0.1 \mu \mathrm{G}$

weaker magnetic fields mean brighter Compton emission for a fixed radio luminosity - and the possibility that the distributions of the magnetic field and nonthermal particles are anticorrelated due to the accumulation of electrons in weak magnetic field regions (Petrosian 2001). The major change between the two figures is an increase in the synchrotron radio emission, which is roughly proportional to the square of the magnetic field. The system with the lower magnetic field has a slightly brighter Compton component.

Light curves at various observing frequencies are shown in Figure 8 using the parameters for our standard case given by Figure 6 with $B=1.0 \mu \mathrm{G}$ and a cluster merger beginning at $z_{i}=0.3$. The light curves of the nonthermal radiation exhibit a common behavior independent of frequency. At early times, the spectral power rises rapidly as the clusters merge. The peak emission occurs when the centers of mass of the two clusters pass at $t_{\text {coll }}$, after which the emission exhibits a slow decay and approaches a plateau at times $t \gtrsim t_{\text {acc }}$ when particle injection has stopped. The rate of decay of the emission increases with radio frequency due to the stronger cooling of the higher energy electrons, so that the decay is slowest at lower frequencies. Synchrotron emission from secondary electrons forms the late-time plateaus at radio energies. This behavior is also apparent for the HXR emission, although it is formed by primary bremsstrahlung and both primary and secondary Compton radiation at late times. At $\gamma$-ray energies, the $\pi^{0}$-decay emission forms a plateau of emission that dominates soon after $t=t_{\text {acc. }}$.

In Figure 9 we show the spectral energy distributions and spectral components for a case where the efficiency $\eta_{p}=10 \%$ for protons and $\eta_{e}=1 \%$ for electrons. An enhancement of the number flux of nonthermal protons compared to electrons is expected in nonrelativistic shock acceleration because protons, unlike electrons, resonate with Alfvén waves at all energies (Baring et al. 1999), and the Larmor radii of protons in the tail of the Maxwellian distribution of the background thermal plasma is greater than that of the electrons, so that protons can more effectively scatter across the shock front. This theoretical expectation is borne out by the large proton-to-electron ratio in the cosmic rays. The enhanced proton contribution produces structure in the $\gamma$-ray spectrum even at $t \approx t_{\text {coll }}$, when the emission is brightest. The $\pi^{0}$-decay feature will be clearly evident in the spectrum of merging clusters of galaxies for such a large proton-to-electron efficiency ratio.

Calculations of the hardest particle injection spectral index $s_{\min }$ formed in cluster merger shocks are shown in Figure 10 as a function of the larger mass $M_{1}$ of the two clusters, with the subcluster mass $M_{2}=10^{14} M_{\odot}$. To derive these results, we used equations in $\S 2.1 .1$ to calculate the dynamics of the clusters, equations in $\S 2.1$.2 to calculate the 

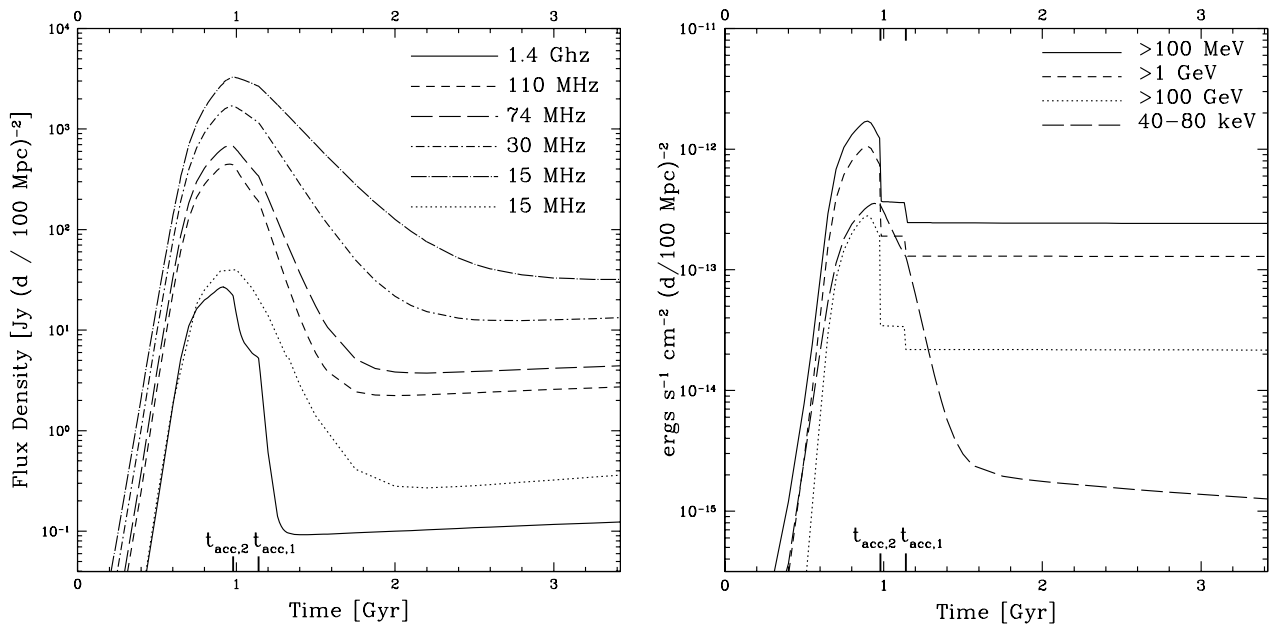

FIG. 8. - Light curves at various observing frequencies produced by a shock formed in a merger between $10^{14}$ and $10^{15} M_{\odot}$ clusters that begins at $z_{i}=0.3$ and is evolved to the present epoch $(t=3.42 \mathrm{Gyr})$. All light curves are for a magnetic field strength of $B=1.0 \mu \mathrm{G}$ unless otherwise noted. Radio light curves in janskys are given at $15 \mathrm{MHz}, 30 \mathrm{MHz}, 74 \mathrm{MHz}, 110 \mathrm{MHz}$, and $1.4 \mathrm{GHz}$ in the left panel, and light curves in energy flux units are given at 40-80 keV, $>100 \mathrm{MeV},>1 \mathrm{GeV}$, and $>100 \mathrm{GeV}$ in the right panel. The $15 \mathrm{MHz}$ light curve is also calculated with a magnetic field strength of $B=0.1 \mu \mathrm{G}($ dotted curve $)$.

sound speed, and equations (27)-(31) to calculate the spectral index from the Mach number. We also assume that the onset of the merger begins at redshift $z_{i}=0.1$; softer injection indexes are obtained for mergers at larger values of $z_{i}$ because of the smaller maximum separations $d$ at earlier times, given by equation (1). We calculate $s_{\min }$ for various values of $r_{c}$ and $\beta$. The values $\left(r_{c}, \beta\right)=(0.05,0.8),(0.05$, $0.45),(0.5,0.8)$, and $(0.5,0.45)$ roughly correspond to the extrema in the range of these parameters measured for 45 X-ray clusters observed with ROSAT (Wu \& Xue 2000). Also shown are values of $s_{\min }$ for $\left(r_{c}, \beta\right)=(0.179,0.619)$, which are the average values of these parameters for the 45 $\mathrm{X}$-ray clusters, and $\left(r_{c}, \beta\right)=(0.25,0.75)$, which are the standard parameters used in the calculations.

The uppermost curves with large $r_{c}$-values display a maximum. At larger values of $M_{1}, s_{\min }$ hardens because of the stronger gravitational potential. Although $s_{\min }$ spans a wide range of values, the value of $s_{\min }$ corresponding to the average values of $r_{c}$ and $\beta$ lies in the range $2.1 \lesssim s_{\min } \lesssim 2.4$, and more realistically in the range $2.2 \lesssim s_{\min } \lesssim 2.4$ because clusters with masses $3 \times 10^{15}$ to $10^{16} M_{\odot}$ are very rare. Because $z_{i}=0.1$, there is a constraint on the available time for the interaction to occur. At low values of $M_{1}$, the gravitational interaction is small so that the interaction takes a longer time to occur. The features in the curves between $10^{14}$ and $10^{14.5} M_{\odot}$ are a result of this effect.

\section{DISCUSSION}

We have presented results of a computer simulation designed to calculate the temporal evolution of nonthermal particles accelerated by a shock front formed between two merging clusters of galaxies. We then calculated the expected total nonthermal photon spectra resulting from synchrotron, bremsstrahlung, and Compton radiation from both primary and secondary electrons and $\pi_{0}$-decay
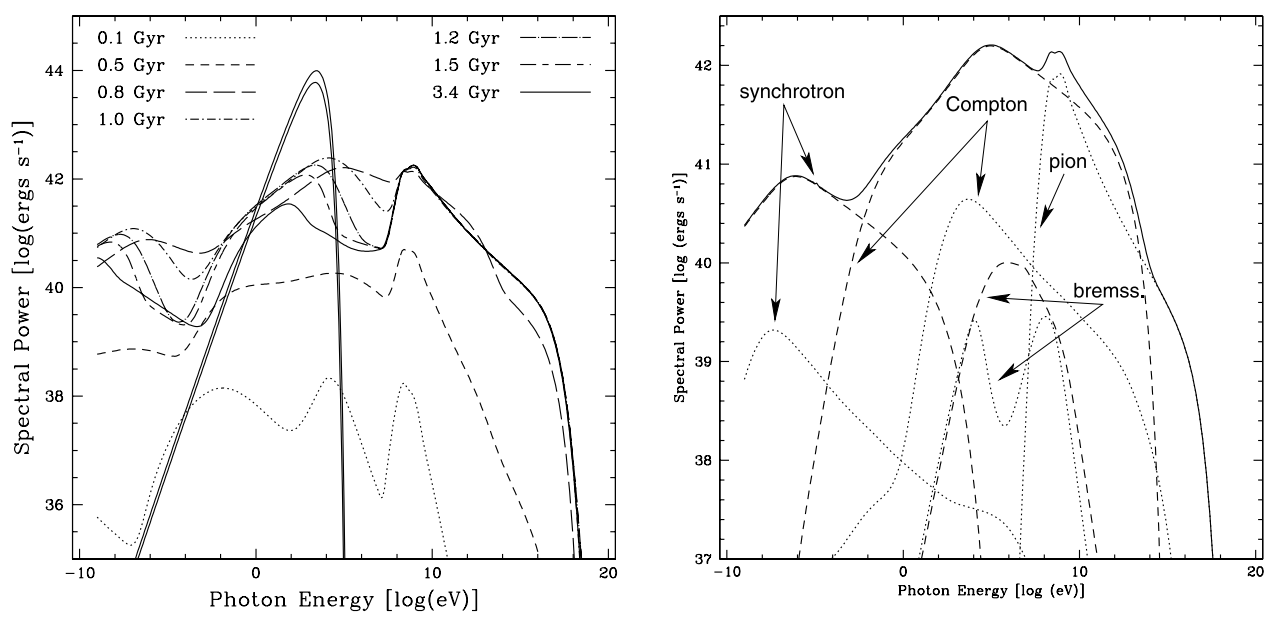

FIG. 9. - Same as the $z_{i}=0.3$ case in Fig. 6, except that $\eta_{e}=1 \%$ and $\eta_{p}=10 \%$. Right panel shows the separate radiation components at time $t=0.8$ Gyr using the same notation as Fig. 5, and the left panel shows the total spectral power at various times. 


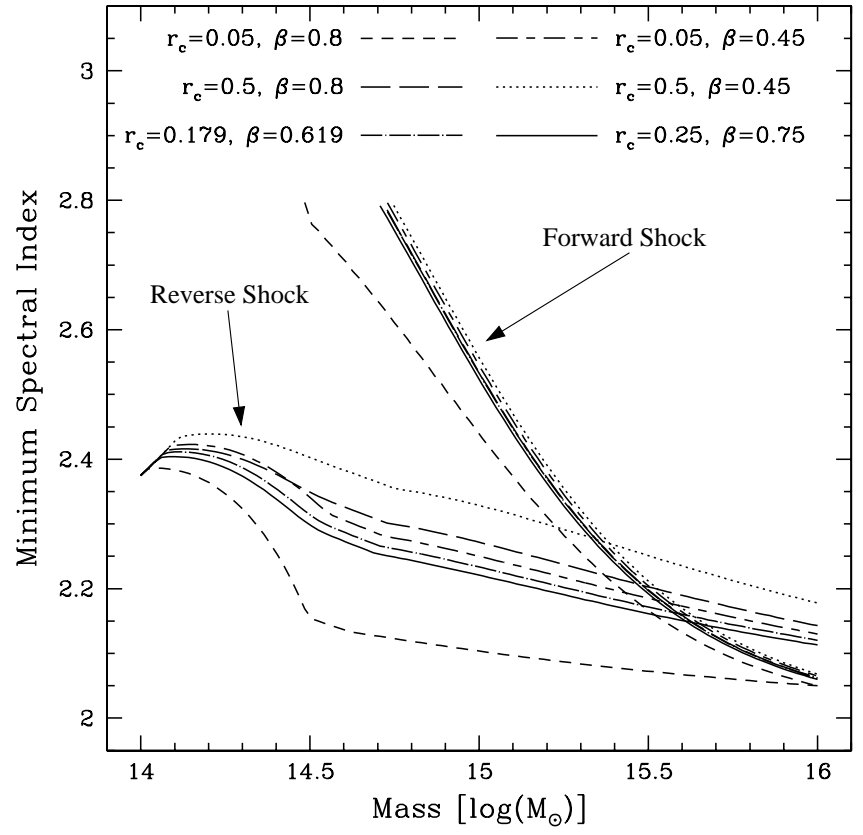

FIG. 10.-Calculations of the hardest particle injection spectral indexes $s_{\min }$ formed in cluster merger shocks as a function of the larger mass $M_{1}$ of the two clusters, for various values of $r_{c}$ and $\beta$. The values of $r_{c}$ are given in Mpc. The minimum spectral index for the forward and reverse shock is shown in the figure. The mass of the subcluster is $M_{2}=10^{14} M_{\odot}$.

$\gamma$-radiation from accelerated protons. Our results apply to the shocks formed in the merger of two clusters of galaxies and not to the accretion shocks formed by spherical infall of matter during the process of structure formation. Theoretical studies suggest that accretion shocks should exist (Bertschinger 1985; Miniati et al. 2000) and that each cluster itself will be the center of its own accretion flow. Although X-ray and radio observations (Kassim et al. 2001; Markevitch \& Vikhlinin 2001; Fusco-Femiano et al. 2003b; Bagchi 2003; Valtchanov et al. 2002) of halos and relics provide strong evidence for the existence of merger shocks, there is also observational evidence for accretion shocks (Ensslin et al. 1998, 2001; Slee et al. 2001; Ensslin \& Brüggen 2002). We have focused on merger shocks in this study. Previous work by Fujita \& Sarazin (2001) would classify our simulations as accretion events, and they showed the luminosity of these accretion shocks to be $\sim 10^{43} \mathrm{ergs} \mathrm{s}^{-1}$ when $M_{1}=1 \times 10^{15} M_{\odot}$, which is consistent with the results of our simulations.

Beppo-SAX and Rossi X-Ray Timing Explorer observations show the presence of a HXR excess at energies $\gtrsim 20$ keV from A1656 (Coma) (Fusco-Femiano et al. 1999; Rephaeli et al. 1999), A2256 (Fusco-Femiano et al. 2000), and A3667 (Fusco-Femiano et al. 2003a). Our simulations support a Compton-scattering CMB interpretation of the HXR (Rephaeli 1979) and EUV (Hwang 1997; Ensslin \& Biermann 1998; Lieu et al. 1999b; Atoyan \& Völk 2000) emission. As shown in Figures 6 and 7, the nonthermal radiation from the merger shock during the postcollision evolution $\left(>t_{\text {coll }}\right)$ shows an excess above the thermal bremsstrahlung in both the HXR and EUV regions of the electromagnetic spectrum. For the standard parameters used, the calculated luminosity of the nonthermal component is $L_{\mathrm{NT}} \approx 10^{43}$ for the HXR emission between the 20 and $80 \mathrm{keV}$ energies. For the Coma Cluster, BeppoSAX observations indicate that the HXR flux is $2.2 \times$ $10^{-11}$ ergs $\mathrm{cm}^{-2} \mathrm{~s}^{-1}$, which corresponds to an observed luminosity of $\sim 10^{43} \mathrm{ergs} \mathrm{s}^{-1}$ in the $20-80 \mathrm{keV}$. Our simulations for the merger model between the times $t_{\text {coll }}$ and $t_{\text {acc }}$ are consistent with this observed value. Our model does match well the calculated mass ratio of the Coma Cluster merger model (Colless \& Dunn 1996). In A2256, the observed masses of the merging clusters are $1.6 \times 10^{15}$ and $5.1 \times 10^{14} M_{\odot}$ (Berrington et al. 2002). The observed HXR flux is $1.2 \times 10^{-11} \mathrm{ergs} \mathrm{cm}^{-2} \mathrm{~s}^{-1}$, which corresponds to an HXR luminosity $\sim 10^{44} \mathrm{ergs} \mathrm{s}^{-1}$ (Fusco-Femiano et al. 2000). By scaling our models to the observed masses of the merging clusters, the expected HXR luminosity is $\sim 10^{44}$ ergs $\mathrm{s}^{-1}$, which is consistent with the observed values seen in A2256.

The Extreme Ultraviolet Explorer (EUVE) has detected EUV emission in the $\sim 60-250 \mathrm{eV}$ band for a number of clusters. These clusters include Virgo, Coma, Fornax, A2199, A1795, and A4059 (Lieu et al. 1996a, 1996b, 1999a, 1999b; Berghöfer, Bowyer, \& Korpela 2000b; Bowyer, Lampton, \& Lieu 1996; Bowyer \& Berghöfer 1998; Mittaz et al. 1998; Bonamente, Lieu, \& Mittaz 2001; Kaastra et al. 1999). Note, however, that no detection of EUV emission, other than that which could be attributed to thermal tail X-ray emission, is reported from analysis of EUVE observations for Fornax (Bowyer, Korpela, \& Berghöfer 2001), A2199 and A1795 (Berghöfer \& Bowyer 2002), and A4059 (Berghöfer, Bowyer, \& Korpela 2000a). The measured EUV excess in the $60-250 \mathrm{eV}$ band for Coma and A1795 is $2 \times 10^{43} \mathrm{ergs} \mathrm{s}^{-1}$ (Lieu et al. 1996a) and $3 \times 10^{43} \mathrm{ergs} \mathrm{s}^{-1}$, respectively (Bonamente et al. 2001). The calculated EUV luminosity of the nonthermal component from our simulations is $L_{\mathrm{NT}} \approx 10^{43}$ for these EUV energies and is consistent with both clusters. As mentioned before, our simulations are a good match to the Coma Cluster merger scenario. The masses of the merging clusters in A1795 are uncertain (Oegerle \& Hill 1994), and confirming the results of our simulations with the observed luminosity of the EUV excess of A1795 is difficult. An EUV excess of luminosity $\sim 6 \times 10^{42}$ ergs s$^{-1}$ was detected from the cluster A2199 (Kaastra et al. 1999). However, A2199 lacks strong evidence for a current merger event. If any mergers occurred in the formation history of A2199, they occurred long enough in the past to allow the cluster to revirialize. It is possible that an adjacent cluster A2197 is beginning to interact with A2199 (Oegerle \& Hill 2001), but seems unlikely that it is the origin of the EUV excess in the central $400 \mathrm{kpc}$ of the cluster. Depending on the masses of the merging clusters, the decreased luminosity of the EUV emission in A2199 is consistent with a cooling nonthermal particle population. Radio and X-ray observations of the central regions of A2199 indicate it is complex (Owen \& Eilek 1998), and it is possible other mechanisms are producing the EUV excess emission.

We treated in detail the merger of a $10^{14} M_{\odot}$ cluster with a $10^{15} M_{\odot}$ cluster having values of $r_{c}$ and $\beta$ in equation (3) approximately equal to the mean values found in a survey of ROSAT clusters of galaxies (Wu \& Xue 2000). We found that the hardest spectral index reached by the merger shock in this system is $s_{\min } \approx 2.2$ for $z_{i}=0.1$. Because $r_{c}$ and $\beta$ are not correlated with cluster temperature $T_{\mathrm{X}}$ and therefore cluster mass, as can easily be seen from the results of $\mathrm{Wu} \&$ Xue (2000), our model results are reasonably representative of typical observed cluster mergers. 
We consider the detectability of nonthermal synchrotron emission with the Low Frequency Array (LOFAR). ${ }^{2}$ LOFAR is a multielement interferometric low-frequency radio telescope designed for high-resolution radio imaging in the $10-300 \mathrm{MHz}$ frequency range. Figure 8 includes several frequencies observable by LOFAR showing the optimization of the LOFAR telescope for detecting these objects. Estimated sensitivities for the LOFAR telescope range from $1.6 \mathrm{mJy}$ at $15 \mathrm{MHz}$ to $40 \mu \mathrm{Jy}$ at $110 \mathrm{MHz}$. The quoted sensitivities are for $1 \mathrm{hr}$ exposures for a single beam for a total of 13,365 dual-polarization dipoles optimized in the 10-90 $\mathrm{MHz}$ and 213,840 dual-polarization dipoles optimized for the 110-220 MHz, each with a maximum baseline of 400 $\mathrm{km}$. With station diameters of $65 \mathrm{~m}$, the resolution of LOFAR is approximately $12^{\prime \prime}$ at $15 \mathrm{MHz}$ and 2".4 at 110 $\mathrm{MHz}$ at the full extent of the array. Because multielement interferometric radio telescopes have trouble detecting large, extended sources, we must consider the angular size of the shock front. Given a size scale of $\sim 1 \mathrm{Mpc}$, these features will easily be resolved at all frequencies visible to LOFAR at a distance of $100 \mathrm{Mpc}$. As a worst-case scenario, we consider the extent of the array that is only capable of observing the shock front as a point object. For all but the most distant shock fronts and the lowest frequencies, the central $2 \mathrm{~km}$ of the LOFAR array, known as the virtual core, is the optimum configuration. Within this virtual core is found $25 \%$ of the total collection area of the LOFAR array. Approximate peak luminosities taken from Figure 8 are $4 \times 10^{3} \mathrm{Jy}$ at $15 \mathrm{MHz}$ and $5 \times 10^{2} \mathrm{Jy}$ at $110 \mathrm{MHz}$. For a shock front with an angular size of $\approx 30^{\prime}$, this gives $\sim 1 \mathrm{mJy}$ $\operatorname{arcsec}^{-2}$ and $\sim 100 \mu \mathrm{Jy} \operatorname{arcsec}^{-2}$, respectively. At these intensities, LOFAR will be able to detect any cluster merger shocks out to a distance of $\sim 2000 \mathrm{Mpc}$ in the $15 \mathrm{MHz}$ frequency band and $\sim 700 \mathrm{Mpc}$ in the $110 \mathrm{MHz}$ frequency band. These calculations neglect any absorption due to the interstellar medium within the Milky Way.

We use results derived for our standard system to estimate the detectability of cluster mergers with EGRET and GLAST. ${ }^{3}$ Generalizing the approach of Dermer \& Schlickeiser (2002) to extended sources, the number of background counts $N_{\mathrm{bg}}$ from extragalactic diffuse $\gamma$-ray background photons with energies greater than $E_{\gamma}$ detected within solid angle $\Delta \Omega$ of a high-latitude source is given by

$$
N_{\mathrm{bg}}\left(>E_{\gamma}\right) \cong \frac{(\Delta \Omega) A_{0}(\Delta t) K_{B} E_{100}}{\alpha_{B}-a_{0}-1}\left(\frac{E_{\gamma}}{E_{100}}\right)^{1+a_{0}-\alpha_{B}},
$$

where $E_{100}=100 \mathrm{MeV}, K_{B}=(1.72 \pm 0.08) \times 10^{-7}$ photons $\mathrm{cm}^{-2} \mathrm{~s}^{-1} \mathrm{MeV}^{-1}$, and $\alpha_{B}=(2.10 \pm 0.03)$ (Sreekumar et al. 1998). The on-axis effective area of the telescope is approximated by the expression $A_{0}\left(E_{\gamma} / E_{100}\right)^{a_{0}}$, and $\Delta t$ is the exposure-corrected time on-source.

The number of source counts produced by photons with energies greater than $E_{\gamma}$ and detected within solid angle $\Delta \Omega$ centered on the direction to a source is given by

$$
\begin{aligned}
N_{s}\left(>E_{\gamma}\right) \cong & (\Delta t) A_{0} \int_{E_{\gamma}}^{\infty} d E \phi_{s}(E)\left(\frac{E}{E_{100}}\right)^{a_{0}} \\
& \times\left[1-\exp \left(\frac{-\Delta \Omega}{\pi \theta_{d}^{2}(E)+\pi \theta_{s}^{2}}\right)\right] .
\end{aligned}
$$

\footnotetext{
${ }^{2}$ See http://www.lofar.org.

${ }^{3}$ See http://glast.gsfc.nasa.gov.
}

Here $\theta_{d}(E)$ is the energy-dependent angular resolution (point spread function) of the telescope, $\theta_{s}$ is the angular radius of the source, assumed to be energy-independent, and $\phi_{s}(E)$ is the source flux, which we write as $\phi_{s}(E)=\left(\phi_{0} / E_{100}\right)\left(E / E_{100}\right)^{-\alpha_{s}}$ (photons $\mathrm{cm}^{-2} \mathrm{~s}^{-1} \mathrm{MeV}^{-1}$ ), with $E$ in units of $\mathrm{MeV}$. Writing the $\nu L_{\nu}$ spectral power as $\nu L_{\nu}=L_{0}\left(E^{\prime} / E_{100}^{\prime}\right)^{\alpha_{\nu}}, \quad$ where $\quad E^{\prime}=(1+z) E, \quad E_{100}^{\prime}=$ $(1+z) E_{100}$, and $\alpha_{\nu}=2-\alpha_{s}$ is the $\nu L_{\nu}$ source spectral index, we have

$$
\begin{aligned}
\phi_{S}(E) & =\frac{L_{0}}{4 \pi d_{L}^{2}\left(1.6 \times 10^{-6} \operatorname{ergs~} \mathrm{MeV}^{-1}\right) E_{100}^{2}}\left(\frac{E}{E_{100}}\right)^{\alpha_{\nu}-2} \\
& \cong \frac{5.2 \times 10^{-10} L_{43}}{d_{100}^{2}}\left(\frac{E}{E_{100}}\right)^{\alpha_{\nu}-2} .
\end{aligned}
$$

The luminosity distance $d_{L}=100 d_{100} \mathrm{Mpc}$, and the brightest luminosity reached at $100 \mathrm{MeV}$ is $L_{0}=10^{43} L_{43} \mathrm{ergs} \mathrm{s}^{-1}$, noting from the calculations in Figures 6 and 7 that $L_{43} \approx 1$.

The maximum sensitivity occurs when $\Delta \Omega \cong$ $\pi \theta_{d}^{2}(E) \approx \pi \theta_{s}^{2}$, giving

$N_{s}\left(>E_{\gamma}\right) \cong 3.3 \times 10^{-8} \frac{A_{0}\left(\mathrm{~cm}^{2}\right) \Delta t(\mathrm{~s}) L_{43}}{\left(1-a_{0}-\alpha_{\nu}\right)}\left(\frac{E_{\gamma}}{E_{100}}\right)^{1+a_{0}-\alpha_{B}}$.

Writing $\Delta \Omega=\pi \theta^{2}=9.6 \times 10^{-4} \theta^{2}\left({ }^{\circ}\right)$, where $\theta\left(^{\circ}\right)$ is the telescope's acceptance angle in degrees, the background count rate

$N_{\mathrm{bg}}\left(>E_{\gamma}\right) \cong 1.65 \times 10^{-8} \frac{\theta^{2}\left({ }^{\circ}\right) A_{0}\left(\mathrm{~cm}^{2}\right) \Delta t(\mathrm{~s})}{1.1-a_{0}}\left(\frac{E_{\gamma}}{E_{100}}\right)^{a_{0}-1.1}$.

The sensitivity of detection at significance $n_{\sigma}$ is therefore given by

$$
\begin{aligned}
n_{\sigma} & =\frac{N_{s}}{\sqrt{N_{\mathrm{bg}}}} \\
& \cong 2.6 \times 10^{-4} \frac{L_{43}}{d_{100}^{2}} \frac{\sqrt{A_{0}\left(\mathrm{~cm}^{2}\right) \Delta t(\mathrm{~s})}}{\theta\left(^{\circ}\right)\left(1-a_{0}-\alpha_{\nu}\right)}\left(\frac{E_{\gamma}}{E_{100}}\right)^{\alpha_{\nu}+\left(a_{0} / 2\right)-0.45} .
\end{aligned}
$$

High-confidence identification of a source requires that $N_{s} \gg 1$ and $n_{\sigma} \gtrsim 5$.

For the EGRET telescope on the Compton Gamma Ray Observatory, $A_{0} \cong 1200 \mathrm{~cm}^{2}, a_{0} \cong 0$, and a nominal observing time is $\Delta t=10^{6} t_{6} \mathrm{~s}$, with $t_{6} \cong 1$. A source with a radius of $0.5 \mathrm{Mpc}$ will have an angular radius $\theta_{s} \sim 0.3 / d_{100}$. The point-spread function of EGRET is $\theta_{d}(E) \cong 5^{\circ}\left(E / E_{100}\right)^{1 / 2}$, which is larger than the source angular size except for the nearest sources or when observing at the highest energies, in which case the number of sources photons is small. Substituting into equations (36) and (37) gives

$$
\begin{gathered}
N_{s}\left(>E_{\gamma}\right) \simeq 40 t_{6} L_{43}\left(E_{\gamma} / E_{100}\right)^{\alpha_{\nu}-1} /\left[\left(1-\alpha_{\nu}\right) d_{100}^{2}\right], \\
n_{\sigma} \simeq 1.9 \sqrt{t_{6}} L_{43}\left(E_{\gamma} / E_{100}\right)^{\alpha_{\nu}+0.05} /\left[\left(1-\alpha_{\nu}\right) d_{100}^{2}\right] .
\end{gathered}
$$

Unless $L_{43} \gg 1$, we conclude that it is unlikely that EGRET could have detected nonthermal emission from merging clusters of galaxies. 
This is in disagreement with the estimate of Totani \& Kitayama (2000), who argue that a large fraction of the isotropic unidentified EGRET sources could be due to the radiation from merging clusters of galaxies. Totani \& Kitayama (2000) make very optimistic assumptions about the hardness of the spectral index of injected nonthermal electrons and in fact claim that the merger shocks have such large Mach numbers that the injection index is nearly equal to 2.0. As shown in Figure 10, such hard spectral indexes are possible only in the most centrally peaked clusters, but the average cluster merger shock as inferred from observations of galaxy clusters has a minimum injection index between 2.2 and 2.3. Because the energies of electrons that Compton scatter the $\mathrm{CMB}$ radiation to greater than $100 \mathrm{MeV}$ energies exceed $\sim 200 \mathrm{GeV}$, the steeper injection spectrum reduces the available power in these electrons by $1-2$ orders of magnitude, thus accounting for the difference between our expectations and the claims of Totani \& Kitayama (2000), Kawasaki \& Totani (2002), and Colafrancesco (2001). Although it is possible that a few of the unidentified EGRET sources are associated with merging clusters of galaxies, these would involve the less frequent events involving collisions of clusters with masses near $10^{15} M_{\odot}$ and those with hard spectral indexes or dark matter density profiles with strong central peaks.

We have not taken into account effects of a merger tree on the evolution of the nonthermal particle distribution (Gabici \& Blasi 2003). The protons that radiate in the EGRET and GLAST ranges will accumulate and could contribute factors of 2-4 enhancements because of successive mergers, but not enough to significantly alter our conclusions. Our predictions are in accord with a recent analysis of EGRET data (Reimer et al. 2003; see, however, Scharf \& Mukherjee 2002) that fails to detect $\gamma$-ray emission from galaxy clusters that emit the most luminous thermal X-ray emission.

For the Large Area Detector on $G L A S T, A_{0} \cong 6200 \mathrm{~cm}^{2}$, $a_{0} \cong 0.16$, and the on-source observing time for a scanning mode lasting $t_{\mathrm{yr}}$ years is $\Delta t \cong 0.2 \times 3.16 \times 10^{7} t_{\mathrm{yr}} \mathrm{s}$, where the factor 0.2 is the exposure correction. The point-spread function for GLAST is $\theta_{d} \cong 3.5\left(E / E_{100}\right)^{-2 / 3}$, which still generally dominates the source angular size except at the highest energies and for the closest sources. From this we obtain

$$
\begin{gathered}
N_{s}\left(>E_{\gamma}\right) \simeq 1300 t_{\mathrm{yr}} L_{43}\left(E_{\gamma} / E_{100}\right)^{\alpha_{\nu}-0.84} /\left[\left(0.84-\alpha_{\nu}\right) d_{100}^{2}\right], \\
n_{\sigma} \simeq 14 L_{43} \sqrt{t_{\mathrm{yr}}}\left(E_{\gamma} / E_{100}\right)^{\alpha_{\nu}+0.3} /\left[\left(0.84-\alpha_{\nu}\right) d_{100}^{2}\right] .
\end{gathered}
$$

These estimates indicate that clusters of galaxies within $\approx 200 \mathrm{Mpc}$ will be detected with GLAST, but we predict far fewer clusters of galaxies than predicted by Totani \& Kitayama (2000) for the reasons discussed above. Note that the detection significance is only weakly energy-dependent, so that the criterion for detection becomes the requirement of detecting a few photons, which is more easily satisfied at lower energies.

Our results also have relevance to suggestions that clusters of galaxies contribute to the diffuse $\gamma$-ray background. Loeb \& Waxman (2000) have argued that Comptonscattered CMB radiation from relativistic electrons accelerated by shocks formed during structure formation can make a significant contribution to the diffuse extragalactic $\gamma$-ray background, which has a featureless power-law spectrum between a few $\mathrm{MeV}$ and $\sim 100 \mathrm{GeV}$ (Sreekumar et al. 1998). If this emission is provided by cluster merger shocks, then our results indicate that this contribution cannot be significant for two reasons. First, the density distributions inferred from X-ray observations of thermal bremsstrahlung in Abell clusters imply an average minimum injection spectral index that would produce Compton-scattered CMB emission that is softer than the spectrum of the diffuse $\gamma$-ray background. Although those few clusters with the hardest indexes would contribute the most emission above 100 $\mathrm{MeV}$, the superposition of the emission from the softer sources would produce a concave spectrum at lower energies, which is not observed. Second, an associated hadronic signature would be observed even in the case where protons are accelerated with the same efficiency as the electrons at all times except near $t_{\text {coll }}$ (see Figs. 6 and 7). The hadronic feature would, however, be seen in the time-averaged $\gamma$-ray spectrum, so that calculations omitting hadronic emissions are incomplete (Keshet et al. 2003). If, as argued in $\S 3$, the hadronic acceleration efficiency exceeds that of electrons, then a $\pi^{0}$-decay signature should be apparent in the background radiation (see Fig. 9) unless accretion shocks make the dominant source of high-energy $\gamma$-ray cluster emission. In this case, most of the $\gamma$-ray emission should be located on the periphery of the cluster, and the $\pi_{0} \gamma$-rays would be reduced dramatically because of the decreased lower gas density in the proximity of the protons (see Miniati 2003 for a spatially resolved model including both proton and electron emissions).

Should it be the case that cluster merger shocks do produce a large fraction of the diffuse extragalactic $\gamma$-ray background, then both low hadronic efficiencies and dark matter halos with strong central density peaks in the majority of rich clusters would seem to be required (see Fig. 10). Under the assumptions of isothermality assumed by the violent relaxation of dark matter halos that collapse from uniform expansion of the Hubble flow, dark matter halos should not have central density cusps. Furthermore, X-ray temperature and luminosity profiles are inconsistent with strongly peaked central dark matter density profiles (Makino, Sasaki, \& Suto 1998; Wu \& Chiueh 2001) despite the predictions of $N$-body simulations (Navarro, Frenk, \& White 1997). Clusters of galaxies could still make a small contribution of order $\sim 1 \%-10 \%$ to the extragalactic diffuse $\gamma$-ray background radiation (Colafrancesco \& Blasi 1998; Miniati 2002).

Our simulations show that protons may reach $\sim 10^{19} \mathrm{eV}$ for standard parameters but are not accelerated to higher energies, in agreement with the gyroradius estimates of Norman, Melrose, \& Achterberg (1995) for maximum cosmic-ray energies. The maximum energies of protons are primarily constrained by the available acceleration times at early times. At later times, the proton Larmor radii may exceed the size scale of the shock, so that the protons then diffuse into the surrounding galaxy cluster of galaxies and undergo no further acceleration. Competition of the acceleration and $p \gamma$ energy-loss rates plays a small role in limiting the highest energy of protons for our standard parameters.

For a diffusion coefficient corresponding to a Kolmogorov spectrum of turbulence, $\gtrsim 10^{16} \mathrm{eV}$ protons can diffuse from the cluster on a Hubble time (Berezinsky, Blasi, \& Ptuskin 1997). Assuming Bohm diffusion, however, Berezinsky et al. (1997) and Völk, Aharonian, \& Breitschwerdt (1996) showed that clusters of galaxies 
provide a storage room for cosmic rays with energies $\lesssim 10^{18}$ $\mathrm{eV}$ on cosmological timescales. Our calculations, which assume Böhm diffusion, confirm that $\sim 10^{18} \mathrm{eV}$ protons remain within the cluster for times much greater than the lifetime of the shock front (see Fig. 4). For energies $\gtrsim 10^{19}$ $\mathrm{eV}$, the loss of protons from diffusion through the ICM will become significant over the lifetime of the shock front and will become a restriction to the maximum energies. The maximum energy imposed by the Larmor radius size-scale criterion is always less than the maximum energy determined by the diffusion loss timescale for our models. For a given magnetic field, the Larmor radius of nonthermal particles is weakly dependent on the cluster mass $\left(\propto M^{1 / 3}\right)$, and despite the possible range of masses for $M_{1}$, the maximum particle energy $E_{\max } \propto M^{1 / 3}$ and varies little over the mass range of $M_{1}$. Kang et al. (1997) argue that more rapid particle acceleration can take place in perpendicular shocks, but this requires preferential orientations of the magnetic field with respect to the shock direction.

\section{SUMMARY AND CONCLUSIONS}

We have presented the results of a computer code designed to calculate the nonthermal particle distributions of a shock front formed in the merger event of two clusters of galaxies. We have calculated nonthermal particle energy spectra for primary electrons and protons and secondary electrons. Photon spectra were calculated for bremsstrahlung, Compton, and synchrotron processes as well as $\pi^{0}$ decay $\gamma$-radiation from $p$ - $p$ collisions. Our results apply to shocks that form at the interaction boundary between two merging clusters of galaxies and not to cluster accretion shocks that form at the outer regions of the cluster. Evidence for nonthermal particle acceleration in merger shocks, which penetrate into the inner region of the accreting cluster, is provided by radio halos and relics and nonthermal X-ray emission from clusters of galaxies.

The thermal X-ray bremsstrahlung was modeled from observations of luminosities, temperatures, and masses of clusters of galaxies. We modeled the nonthermal emission under the assumption that electrons and protons are accelerated with a $5 \%$ efficiency of the available gravitational energy that is dissipated during the course of gravitational interactions between two merging clusters of galaxies. Particle acceleration at shocks formed between merging clusters of galaxies produces a population of nonthermal electrons that Compton scatters CMB photons. This process naturally produces a power-law distribution of photons in the 40-80 keV energy range with luminosities consistent with HXR observations of the galaxy clusters
A1656, A2256, A3667, and possibly A2199. In addition, the EUV emission observed in A1656 is also consistent with a nonthermal Compton-scattered $\mathrm{CMB}$ origin. Cluster magnetic field strengths $\sim 0.1 \mu \mathrm{G}$ are in accord with this interpretation of the observed HXR excess and EUV emission.

In contrast to the results of Totani \& Kitayama (2000), Kawasaki \& Totani (2002), and Colafrancesco (2001), we have argued that it is unlikely that more than a few of the isotropic unidentified EGRET sources can be attributed to radiation from nonthermal particles produced by cluster merger shocks. Previous studies have assumed hard nonthermal spectra that give brighter $\gamma$-ray emission than implied by our simulation results. Such hard spectra can be obtained only in the infrequent merger events involving two very massive clusters, or between clusters where the dark matter density profiles are centrally peaked.

The diffuse extragalactic $\gamma$-ray background is a featureless power law with photon index of $2.10 \pm 0.03$. The dominant nonthermal $\gamma$-radiation components in cluster merger shocks include Compton-scattered $\mathrm{CMB}$ radiation and bremsstrahlung from nonthermal electrons and $\pi^{0}$-decay emission from nuclear interactions involving nonthermal protons. The $\pi^{0}$-decay signature will be present if the hadronic acceleration efficiency exceeds the electron acceleration efficiency, as expected in diffusive shock acceleration theory. Furthermore, spectra calculated using parameters obtained from ROSAT observations of 45 Abell clusters (Wu \& Xue 2000) produce spectra with slopes $\approx 2.2-2.4$. Unless dark matter density profiles are centrally peaked and hadronic acceleration efficiency is low, our results imply that nonthermal emission from merging clusters of galaxies can make only a minor contribution to the diffuse extragalactic $\gamma$-ray background. Using standard diffusive shock acceleration with mean ICM magnetic fields $\lesssim 1 \mu \mathrm{G}$, our results also indicate that the merger shocks in clusters of galaxies do not accelerate $\gtrsim 10^{19} \mathrm{eV}$ cosmic rays, although additional effects, such as shock obliquity and the presence of preexisting particle populations, could permit higher energy acceleration by these shocks.

We wish to thank Armen Atoyan, Namir Kassim, and Paul Ray for useful discussions and the referee for a detailed and constructive report. We would also like to thank Steve Sturner for providing his supernova code, which was used to begin this project. This work is supported by the Office of Naval Research, NASA Astrophysical Theory Grant DPR S-13756-G, and NASA GLAST Science Investigation Grant DPR S-15634-Y.

\section{APPENDIX A}

\section{PARTICLE ENERGY-LOSS RATES}

\section{A1. ELECTRON LOSS RATES}

Nonthermal electrons lose energy through Coulomb or ionization interactions with background thermal electrons. Energy is lost through this process at the rate

$$
\dot{K}_{e, \text { coul }}\left(E_{e}, t\right)=-\eta_{\mathrm{He}}^{e} n_{\mathrm{ICM}}\left[\frac{4 \pi e^{4} \Lambda(t)}{\beta_{e} m_{e} c}\right]\left[\Xi(t)-\frac{d \Xi(t)}{d x}\right],
$$


where we have made use of the following definitions:

$$
\begin{gathered}
\Xi(t)=2 \pi^{-1 / 2} \int_{0}^{x(t)} d y y^{1 / 2} \exp (-y), \\
\Lambda(t)=24-\ln \left[\frac{\left(\eta_{\mathrm{He}}^{e} n_{\mathrm{ICM}}\right)^{1 / 2}}{T_{e}^{\mathrm{eV}}(t)}\right], \\
x(t)=\frac{m_{e} v_{e}^{2}}{2 k T_{e}(t)} .
\end{gathered}
$$

The quantity $n_{\mathrm{ICM}}$ is the proton density of the ICM, $\beta_{e} c$ is the electron velocity, $T_{e}(t)$ is the electron temperature given in kelvins, and $k$ is Boltzmann's constant. Nonthermal electrons diffuse in energy space according to the diffusion coefficient for Coulomb scattering

$$
D_{e, \text { coul }}\left(K_{e}, t\right)=\eta_{\mathrm{He}}^{e} n_{\mathrm{ICM}}\left[\frac{8 \pi e^{4} \Lambda(t)}{\beta_{e} m_{e} c}\right] k T_{e}(t) \Xi(t)
$$

(Miller, Larosa, \& Moore 1996).

For a fully ionized ICM, the bremsstrahlung energy-loss rate (Blumenthal \& Gould 1970) is given by the expression

$$
\dot{K}_{e, \text { brem }}\left(K_{e}, t\right)=-\eta_{\mathrm{He}}^{n} n_{\mathrm{ICM}}\left(\frac{8 e^{6}}{m_{e}^{2} c^{4} \hbar}\right)\left(\ln \gamma_{e}+0.36\right)\left(K_{e}+m_{e} c^{2}\right)
$$

where $\eta_{\mathrm{He}}^{n}=1.3$ is an enhancement factor for Helium that results from the $Z(Z+1)$ dependence of the loss rate. The synchrotron energy-loss rate for electrons averaged over pitch angle in a magnetic field of strength $B$, Lorentz factor $\gamma_{e}$, and velocity $\beta_{e}$ is (Rybicki \& Lightman 1979)

$$
\dot{K}_{e, \mathrm{syn}}\left(E_{e}, t\right)=-\frac{4}{3} c \sigma_{\mathrm{T}} \gamma_{e}^{2} \beta_{e}^{2}\left(\frac{B^{2}}{8 \pi}\right)
$$

where $\sigma_{\mathrm{T}}$ is the Thomson cross section.

Electrons lose energy by Compton-scattering ambient photons in the ICM environment, as described by equations (13), (16), and (17) for the cluster X-ray, stellar, and CMB radiation fields, respectively. The spectral distribution of each radiation field is approximated by a blackbody spectrum of temperature $T_{\mathrm{X}}$ (see eq. [9]), $4000 \mathrm{~K}$, and $2.7(1+z) \mathrm{K}$ at epoch $z$. The Compton energy-loss rate is given by

$$
\dot{K}_{e, \mathrm{comp}}\left(E_{e}, t\right)=-\frac{4}{3} c \sigma_{\mathrm{T}} \gamma_{e}^{2} \beta_{e}^{2} \sum_{i} U_{i} \sigma_{\mathrm{KN}}\left(\gamma_{e} \vartheta_{i}\right)
$$

(Skibo 1993). The quantity $\sigma_{\mathrm{KN}}\left(\gamma_{e}, \vartheta_{i}\right) \equiv \sigma_{\mathrm{C}}\left(\gamma_{e} \vartheta_{i}\right) /\left[\sigma_{\mathrm{T}}\left(1+\gamma_{e} \vartheta_{i}\right)\right]$ is a Klein-Nishina correction factor, where $\vartheta_{i} \equiv k T_{i} / m_{e} c^{2}$ is the dimensionless temperature and $\sigma_{\mathrm{C}}\left(\epsilon_{\gamma}\right)$ is the Compton scattering cross section for photons with energy $\epsilon_{\gamma}=h \nu / m_{e} c^{2}$ in the electron rest frame.

Electrons can also lose energy through the double (or "radiative") Compton process in which a second photon is created during an electron-photon interaction. Estimates by Ram \& Wang (1971) and Gould (1975) show that the ratio of energy-loss rates through double Compton scattering and ordinary Compton scattering is $\sim \frac{1}{4}$ when the typical photon energy in the electron rest frame is $\sim 50 \mathrm{MeV}$. This ratio only reaches unity when the rest-frame photon energy is $\sim 5 \mathrm{TeV}$. Synchrotron energy losses completely dominate at these energies, so the energy-loss rate through double Compton scattering can be safely neglected. A detailed treatment of electron energy losses in galaxy clusters, although at energies where double Compton is not important, is given by Sarazin (1999).

\section{A2. PROTON LOSS RATES}

The Coulomb energy-loss rate for protons is given by

$$
\dot{K}_{p, \text { coul }}\left(K_{p}, t\right)=-\eta^{e} n_{\mathrm{ICM}}\left[\frac{4 \pi e^{4} \Lambda(t)}{\beta_{p} m_{p} c}\right]\left[\left(\frac{m_{p}}{m_{e}}\right) \Xi(t)-\frac{d \Xi(t)}{d x}\right] .
$$

In addition to energy losses, the protons diffuse in energy space according to the diffusion coefficient

$$
D_{p, \text { coul }}\left(K_{p}, t\right)=\eta^{e} n_{\mathrm{ICM}}\left[\frac{8 \pi e^{4} \Lambda(t)}{\beta_{p} m_{p} c}\right] k T_{e}(t) \Xi(t)
$$

(Huba 1994). 
Protons undergo proton-proton collisions to produce pions in which a proton loses $\approx \frac{1}{3}$ of its energy per collision. After three collisions, the proton is assumed to be lost from the system. The timescale for this loss is

$$
\tau_{\text {pion }}\left(E_{p}, t\right)=\frac{1}{c \beta_{p} n_{\mathrm{ICM}}\left(\sigma_{\mathrm{H}}+0.1 \sigma_{\mathrm{He}}\right)}
$$

(Sturner et al. 1997), where $\sigma_{\mathrm{H}}$ and $\sigma_{\mathrm{He}}$ are the inelastic cross sections for pion-producing collisions with protons and helium, respectively. Measured values for these cross sections are $\sigma_{\mathrm{H}}=28$ mbarns and $\sigma_{\mathrm{He}}=100$ mbarns, well above threshold (Meyer 1972).

High-energy protons interact with the CMB photons because of photomeson production. It requires about five interactions for protons to lose a substantial fraction of their energy because of interactions with photons with proton rest-frame energies in the range 200-500 MeV, and about two interactions for photons whose energies in the rest frame of the protons are greater than $500 \mathrm{MeV}$. The timescale for the catastrophic loss is given by

$$
\tau_{p \gamma \rightarrow \pi}^{-1}\left(E_{p}, t\right)=\frac{c}{2} \int_{0}^{\infty} d \epsilon n_{\gamma}(\epsilon) \int_{-1}^{1} d \mu(1-\mu) k_{p \gamma}\left(\epsilon^{\prime}\right) \sigma_{p \gamma \rightarrow \pi}\left(\epsilon^{\prime}\right),
$$

where $\sigma_{p \gamma \rightarrow \pi}\left(\epsilon^{\prime}\right)$ is the cross section of the proton-photon interaction and $\epsilon^{\prime}=\gamma_{p} \epsilon(1-\mu)$ is the photon energy in the rest frame of the proton. Here $\mu=\cos \theta$, and $\theta$ is the angle between the directions of the interacting photon and proton. We let $\sigma_{p \gamma \rightarrow \pi}\left(\epsilon^{\prime}\right)=380$ and $120 \mu$ barns, and the inelasticity coefficient $k_{p \gamma}=0.2$ and 0.6 , for $200 \mathrm{MeV} \leq \epsilon^{\prime} \leq 500 \mathrm{MeV}$ and $\epsilon^{\prime}>500 \mathrm{MeV}$, respectively (Atoyan \& Dermer 2001). The number density of CMB photons at redshift $z$ is denoted by $n_{\gamma}(\epsilon)$.

Protons diffuse through the ICM at a rate that depends on the diffusion coefficient. This diffusion process is a random walk that allows particles to escape from the system. Protons that escape from the cluster merger are treated as a catastrophic loss with timescale (Völk et al. 1996)

$$
\tau_{d}\left(E_{p}\right) \approx \frac{R_{1}^{2}}{\kappa_{\mathrm{Bohm}}\left(E_{p}\right)}
$$

where we employ the Böhm diffusion coefficient $\kappa_{\mathrm{Bohm}}\left(E_{p}\right)=\beta c r_{\mathrm{L}} / 3$ for particle diffusion, and $r_{\mathrm{L}}$ is the particle Larmor radius.

\section{A3. PION PRODUCTION FROM $p-p$ COLLISIONS}

Nonthermal protons collide with ambient gas particles to produce secondaries through several channels. The five dominant channels are (1) $p+p \rightarrow \pi^{0}+X$, (2) $p+p \rightarrow \pi^{+}+X$, (3) $p+p \rightarrow \pi^{-}+X$, (4) $p+p \rightarrow K^{+}+X$, and (5) $p+p \rightarrow K^{-}+X$. Here $X$ refers to any by-products produced in the reaction other than the particle indicated. It is also understood that channel 2 does not include the contribution resulting from the production of deuterium in the reaction $p+p \rightarrow \pi^{+}+d$, which can be treated separately but is a small contribution to the total secondary production. The secondary electron production is dominated by the first two pion channels, and these are the only two channels considered in our calculations. Our calculation technique follows the method described by Dermer (1986a, 1986b) and Moskalenko \& Strong (1998); see also Blasi \& Colafrancesco (1999). This model combines the isobaric model (Stecker 1970) at energies less than $3 \mathrm{GeV}$ and the scaling model (Badhwar, Golden, \& Stephens 1977) for energies greater than $7 \mathrm{GeV}$. We use a linear combination to join the two models in the transition region between 3 and $7 \mathrm{GeV}$.

For proton energies less than $3 \mathrm{GeV}$, the production of pions produced by proton-proton collisions is mediated by the excitation of a $\Delta_{3 / 2}$ isobar. Assuming the outgoing $\Delta_{3 / 2}$ isobar of mass $m_{\Delta}$ is collinear with the initial direction of the colliding protons in the center-of-momentum system (CM) with isotropically distributed decay products in the laboratory system (LS), the pion distribution in the LS of the isobaric model is given by

$$
f\left(K_{\pi} ; K_{p}, m_{\Delta}\right)=\frac{1}{4 m_{\pi} \gamma_{\pi}^{\prime} \beta_{\pi}^{\prime} \gamma_{\Delta}^{+} \beta_{\Delta}^{+}}\left\{H\left[\gamma_{\pi} ; \aleph^{+}(-), \aleph^{+}(+)\right]+\frac{\gamma_{\Delta}^{+} \beta_{\Delta}^{+}}{\gamma_{\Delta}^{-} \beta_{\Delta}^{-}} H\left[\gamma_{\pi} ; \aleph^{-}(-), \aleph^{-}(+)\right]\right\},
$$

where the Heaviside function $H[x ; a, b]$ is defined to be $=1$ if $a \leq x \leq b$, otherwise $=0$. The function $\aleph^{ \pm}(\mp)=\gamma_{\Delta}^{ \pm} \gamma_{\pi}^{\prime}\left(1 \mp \beta_{\Delta}^{ \pm} \beta_{\pi}^{\prime}\right)$, and $T_{\pi}$ is the pion LS kinetic energy. The forward (+) and backward (-) moving isobar Lorentz factors are $\gamma_{\Delta}^{ \pm}=\gamma_{c} \gamma_{\Delta}^{*}\left(1 \pm \beta_{c} \beta_{\Delta}^{*}\right)$, where $\gamma_{c}=\sqrt{s} / 2 m_{p}$ is the Lorentz factor of the CM with respect to the LS, and $\gamma_{\Delta}^{*}=\left(s+m_{\Delta}^{2}+m_{\pi}^{2}\right) / 2 s^{1 / 2} m_{\Delta}$ is the Lorentz factor of the isobar in the CM. The pion Lorentz factor in the rest frame of the $\Delta$ isobar is $\gamma_{\pi}^{\prime}=\left(m_{\Delta}^{2}+m_{\pi}^{2}-m_{p}^{2}\right) / 2 m_{\Delta} m_{\pi}$.

The pion spectrum resulting from the $p$ - $p$ collisions is calculated by evaluating the following integral over the isobar mass spectrum:

$$
\frac{d N\left(K_{\pi}, K_{p}\right)}{d T_{\pi}}=w_{r}\left(K_{p}\right) \int_{m_{p}+m_{\pi}}^{\sqrt{s}-m_{p}} d m_{\Delta} B_{\mathscr{W}}\left(m_{\Delta}\right) f\left(K_{\pi} ; K_{p}, m_{\Delta}\right)
$$


The term $B_{\mathscr{W}}\left(m_{\Delta}\right)$ is the normalized isobar mass spectrum given by the Breit-Wigner distribution

$$
B_{\mathscr{W}}\left(m_{\Delta}\right)=\frac{w_{r}\left(K_{p}\right) \Gamma}{\left(m_{\Delta}-\left\langle m_{\Delta}\right\rangle\right)^{2}+\Gamma^{2}}
$$

with average isobar mass $\left\langle m_{\Delta}\right\rangle$, and normalization factor

$$
w_{r}\left(K_{p}\right)=\left[\arctan \left(\frac{\sqrt{s}-m_{p}-\left\langle m_{\Delta}\right\rangle}{\Gamma}\right)-\arctan \left(\frac{m_{p}+m_{\pi}-\left\langle m_{\Delta}\right\rangle}{\Gamma}\right)\right]^{-1} .
$$

Our calculations only consider the $\Delta_{3 / 2}(1232)$ isobar, which has a resonance width $\Gamma=\frac{1}{2} \times 115 \mathrm{MeV}$ (Particle Data Group 1984).

For protons whose energies are greater than $7 \mathrm{GeV}$, we adopt the scaling model of Stephens \& Badhwar (1981). The Lorentz invariant cross section given by Badhwar et al. (1977) and Stephens \& Badhwar (1981) for neutral and charged pion production is given by

$$
E_{\pi} \frac{d^{3} \sigma}{d^{3} p_{\pi}}=\mathscr{A} \mathscr{G}_{\pi}\left(E_{p}\right)\left(1-\zeta_{\pi}\right)^{2} \exp \left(\frac{-\mathscr{B} p_{\perp}}{1+4 m_{p}^{2} / s}\right)
$$

where we have made use of the following definitions:

$$
\begin{gathered}
\mathscr{G}_{\pi^{ \pm}}\left(E_{p}\right)=\left(1+\frac{4 m_{p}^{2}}{s}\right)^{\mathscr{R}}, \\
\mathscr{G}_{\pi^{0}}\left(E_{p}\right)=\left(1+23 E_{p}^{-2.6}\right)\left(1-\frac{4 m_{p}^{2}}{s}\right)^{\mathscr{R}}, \\
\mathscr{Q}=\frac{C_{1}-C_{2} p_{\perp}+C_{3} p_{\perp}^{2}}{\sqrt{1+4 m_{p}^{2} / s}}, \\
\zeta_{\pi}=\sqrt{x_{\|}^{*}+\left(\frac{4}{s}\right)\left(p_{\perp}^{2}+m_{\pi}^{2}\right)}, \\
x_{\|}^{*}=\frac{2 m_{\pi} \gamma_{\pi} \gamma_{c} s^{1 / 2}\left(\beta_{\pi} \cos \theta-\beta_{c}\right)}{\sqrt{\left(s-m_{\pi}^{2}-m_{X}^{2}\right)^{2}-4 m_{\pi}^{2} m_{X}^{2}}} .
\end{gathered}
$$

The constants $\mathscr{A}, \mathscr{B}, C_{1,2,3}$, and $\mathscr{R}$ are given by Badhwar et al. (1977) and Stephens \& Badhwar (1981), and $\theta$ is the pion LS polar angle. Adopted values for the constants $\mathscr{A}, \mathscr{B}, C_{1,2,3}$, and $\mathscr{R}$ are given in Table 1 . The quantities $m_{X}$ depends on the channel under consideration and are (1) $m_{X}=2 m_{p}$, (2) $m_{X}=m_{p}+m_{n}$, (3) $m_{X}=2 m_{p}+m_{\pi}$, (4) $m_{X}=m_{p}+m_{n}$, and (5) $m_{X}=2 m_{p}+m_{K}$.

To calculate the LS energy distribution of pions, we integrate the following over the pion LS polar angle:

$$
Q_{\pi}\left(E_{\pi}, E_{p}\right)=\frac{2 \pi p_{\pi}}{\left\langle\eta \sigma_{\pi}\left(E_{p}\right)\right\rangle} \int_{\cos \theta_{\max }}^{1} d \cos \theta\left(E_{\pi} \frac{d^{3} \sigma}{d^{3} p_{\pi}}\right),
$$

\begin{tabular}{|c|c|c|c|}
\hline \multirow[b]{2}{*}{ Constant } & \multicolumn{3}{|c|}{ Channel } \\
\hline & 1 & 2 & 3 \\
\hline 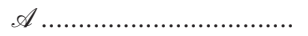 & 140 & 153 & 127 \\
\hline ஜ & 5.43 & 5.55 & 5.3 \\
\hline$C_{1} \ldots \ldots \ldots \ldots \ldots \ldots \ldots$ & 6.1 & 5.3667 & 7.0334 \\
\hline$C_{2} \ldots \ldots \ldots \ldots \ldots \ldots \ldots \ldots$ & -3.3 & -3.5 & -4.5 \\
\hline$C_{3} \ldots \ldots \ldots \ldots \ldots \ldots \ldots \ldots \ldots \ldots \ldots$ & 0.6 & 0.8334 & 1.667 \\
\hline $\mathscr{R}$ & 2 & 1 & 3 \\
\hline
\end{tabular}

where $-1 \leq \cos \theta_{\max } \leq 1$ and is defined as

$$
\cos \theta_{\max }=\frac{1}{\beta_{c} \gamma_{c} p_{\pi}}\left(\gamma_{c} E_{\pi}-\frac{s-m_{X}^{2} m_{\pi}^{2}}{2 \sqrt{s}}\right) .
$$

The quantity $\left\langle\eta \sigma_{\pi}\left(E_{p}\right)\right\rangle$ is the inclusive cross section, and its values are taken from equations (5)-(9) of Dermer (1986a).

TABLE 1

Adopted Values for Constants Defining the Invariant

Cross Section For $p+p \rightarrow X$ Reactions 
The pions will decay to produce muons. These muon are created fully polarized. This results in a $e^{ \pm}$decay asymmetry. The muons created by the pion decay are created in the rest frame of the pion with Lorentz factor $\gamma_{\mu}=\left(m_{\pi}^{2}+m_{\mu}^{2}\right) / 2 m_{\pi} m_{\mu} \approx 1.039$, and $\beta_{\mu} \approx 0.2714$. In the rest frame of the muons, the resulting electron-decay spectrum is given by (Commins 1973; Orth \& Buffington 1976)

$$
\frac{d^{2} N}{d E_{e}^{*} d \cos \theta^{*}}=\frac{2 \epsilon^{2}(e-2 \epsilon)}{m_{\mu}}\left[1+\xi\left(\frac{1-2 \epsilon}{3-2 \epsilon}\right) \cos \theta^{*}\right],
$$

where $E_{e}^{*}$ is the electron energy in the muon rest frame and helps to define the quantity $\epsilon=2 E_{e}^{*} / m_{\mu}$. The angle between the polarization angle of the parent muon and the resulting electron momentum is $\theta^{*}$. The quantity $\xi= \pm 1$ for $\mu^{ \pm} \rightarrow e^{ \pm}$.

For a given electron energy $E_{e}$, the normalized electron-decay energy distribution in the frame where the pions are isotropic is (Dermer 1986a)

$$
\frac{d N\left(E_{e} ; y_{\pi}\right)}{d E_{e}}= \begin{cases}\phi\left(\varsigma_{-}\right)-\phi\left(\varsigma_{+}\right), & \varsigma_{+}<\varsigma_{-}<\omega_{-}, \\ \psi\left(\varsigma_{-}\right)-\psi\left(\omega_{-}\right)+\phi\left(\omega_{-}\right)-\phi\left(\varsigma_{+}\right), & \varsigma_{+}<\omega_{-}<\varsigma_{-}<\omega_{+}, \\ \psi\left(\omega_{+}\right)-\psi\left(\omega_{-}\right)+\phi\left(\omega_{-}\right)-\phi\left(\varsigma_{+}\right), & \varsigma_{+}<\omega_{-}<\omega_{+}<\varsigma_{-}, \\ \psi\left(\varsigma_{-}\right)-\psi\left(\varsigma_{+}\right), & \omega_{-}<\varsigma_{+}<\varsigma_{-}<\omega_{+}, \\ \psi\left(\omega_{+}\right)-\psi\left(\varsigma_{+}\right), & \omega_{-}<\varsigma_{+}<\omega_{+}<\varsigma_{-}, \\ 0, & \omega_{+}<\varsigma_{-},\end{cases}
$$

where we have introduced the following definitions:

$$
\begin{gathered}
\varsigma_{ \pm}=\frac{E_{e}}{\gamma_{\mu} \gamma_{\pi} m_{\mu}\left(1 \pm \beta_{\pi}\right)}, \\
\omega_{ \pm}=\frac{1 \pm \beta_{\mu}}{2}, \\
\phi(x)=\frac{8 \gamma_{\mu}^{5} x^{2}}{d E_{e}^{*} d \cos \theta^{*}}\left[\frac{\left(3-u \beta_{\mu}^{2}\right)\left(1-\beta_{\mu}^{2}\right)}{2}-\frac{4 x\left(3+\beta_{\mu}^{2}-4 u \beta_{\mu}^{2}\right)}{9}\right], \\
\psi(x)=\frac{1}{6 \beta_{\mu} \gamma_{\mu} \beta_{\pi} \gamma_{\pi} m_{\mu}}\left[(5+u) \ln x-\frac{6\left(u+2 u \beta_{\mu}+3\right) x^{2}}{\left(1+\beta_{\mu}\right)^{2}}+\frac{16\left(u+3 u \beta_{\mu}+2\right) x^{3}}{3\left(1+\beta_{\mu}\right)^{3}}\right],
\end{gathered}
$$

where $u=\xi / \beta_{\mu}$, and $\xi=1$ for positrons and -1 for electrons. Figure 11 shows the energy distributions of electrons and positrons formed through $p$ - $p$ collisions by monoenergetic protons with a variety of energies for channels 1 and 2 (compare Murphy, Dermer, \& Ramaty 1987).

The resulting electron or positron spectrum formed by the decay of isotropically produced pions is

$$
Q_{e}\left(E_{e}\right)=\int_{\bar{\gamma}_{\pi}\left(E_{e}\right)}^{\infty} d \gamma_{\pi} Q_{\pi}\left(\gamma_{\pi}\right) \frac{d N\left(E_{e} ; y_{\pi}\right)}{d E_{e}},
$$
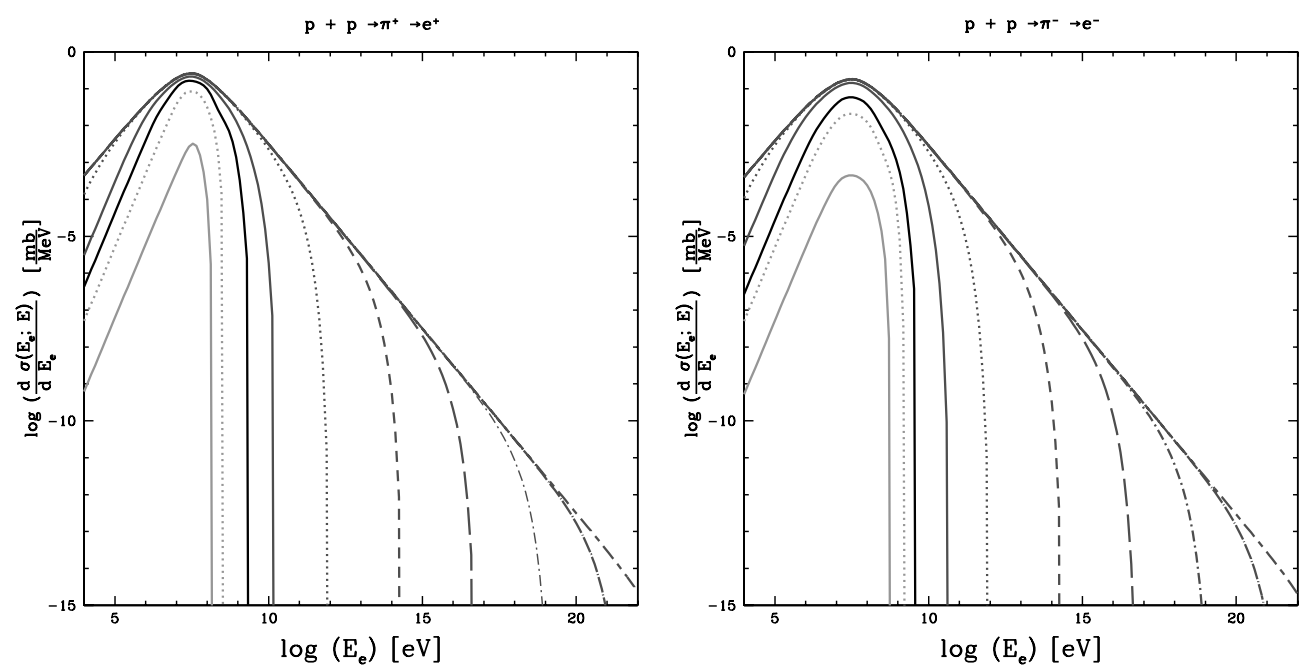

FIG. 11.-Energy distributions of secondary positrons (left) and electrons (right) produced in $p$ - $p$ nuclear collisions by protons with energies $K_{p}=3.16 \times 10^{8}, 5.62 \times 10^{8}, 3.16 \times 10^{9}, 2.15 \times 10^{10}, 1.0 \times 10^{12}, 2.5 \times 10^{14}, 6.3 \times 10^{16}, 1.58 \times 10^{19}, 4.0 \times 10^{21}$, and $1.0 \times 10^{24} \mathrm{eV}$ ( from bottom to top) for the $p+p \rightarrow e^{+}+X$ channel and protons of energies $K_{p}=1.0 \times 10^{9}, 2.15 \times 10^{9}, 5.0 \times 10^{9}, 5.0 \times 10^{10}, 1.0 \times 10^{12}, 2.5 \times 10^{14}, 6.3 \times 10^{16}, 1.58 \times 10^{19}, 4.0 \times 10^{21}$, and $1.0 \times 10^{24} \mathrm{eV}$ (from bottom to top) for the $p+p \rightarrow e^{-}+X$ channel. 
with $Q_{\pi}\left(\gamma_{\pi}\right)$ given by equation (A24) and $\bar{\gamma}_{\pi}=\left(E_{e} / E_{e}^{\max }+E_{e}^{\max } / E_{e}\right) / 2$ if $E_{e}>E_{e}^{\max }=m_{\mu}\left(1+\beta_{\mu}\right) \gamma_{\mu} / 2 \approx 69.8 \mathrm{MeV}$; otherwise, $\bar{\gamma}_{\pi}=1$.

\section{A4. KNOCK-ON ELECTRONS}

Nonthermal protons interact with thermal electrons through Coulomb collisions to produce a population of nonthermal knock-on electrons. The knock-on electron source function in electrons $\mathrm{cm}^{-3} \mathrm{~s}^{-1}$ is given by

$$
Q_{k}\left(\gamma_{e}\right) \cong 1.75 n_{\mathrm{ICM}}(r) 4 \pi \int_{\gamma_{1}}^{\infty} d \gamma_{p} \Phi_{p \rightarrow e}\left(\gamma_{e}, \gamma_{p}\right) \frac{d J_{p}}{d \gamma_{p}}
$$

(Abraham, Brunstein, \& Cline 1966), where the factor 1.75 accounts for the presence of heavier nuclei in the ICM and cosmic rays, $\gamma_{e}$ is the Lorentz factor of the electron, $\gamma_{p}$ is the Lorentz factor of the proton, and $d J_{p} / d \gamma_{p}$ is the differential cosmic-ray proton intensity in particles $\mathrm{cm}^{-2} \mathrm{~s}^{-1} \mathrm{sr}^{-1}$. The differential probability for the production of an electron having a total energy per unit rest-mass energy in $d \gamma_{e}$ at $\gamma_{e}$ by the collision of a cosmic-ray proton of Lorentz factor $\gamma_{p}$ in units of $\mathrm{cm}^{2} \mathrm{~s}^{-1}$ is given by

$$
\Phi_{p \rightarrow e}\left(\gamma_{e}, \gamma_{p}\right) d \gamma_{e}=\frac{2 \pi r_{e}^{2}}{\left(1-\gamma_{p}^{-2}\right)}\left\{\frac{1}{\left(\gamma_{e}-1\right)^{2}}-\frac{m_{e}\left[\gamma_{p}+\left(m_{e}^{2}+m_{p}^{2}\right) /\left(2 m_{p} m_{e}\right)\right]}{m_{p}\left(\gamma_{e}-1\right) \gamma_{p}^{2}}+\frac{m_{e}^{2}}{2 m_{p}^{2} \gamma_{p}^{2}}\right\} d \gamma_{e},
$$

(Abraham et al. 1966), where $r_{e}$ is the classical radius of the electron. The maximum transferable energy is given by

$$
\gamma_{\max }=1+\frac{\gamma_{p}^{2}-1}{\left(m_{e} / m_{p}\right)\left[\gamma_{p}+\left(m_{e}^{2}+m_{p}^{2}\right) /\left(2 m_{e} m_{p}\right)\right]} .
$$

The lower bound of the integration in equation (A30) is determined by solving the inequality $\gamma_{e} \leq \gamma_{\max }$ for $\gamma_{p}$. The result is

$$
\gamma_{p} \geq \gamma_{1}=\frac{m_{p}}{2 m_{e}}\left(\gamma_{e}-1\right)+\left[1+\frac{1}{2}\left(1+\frac{m_{p}^{2}}{m_{e}^{2}}\right)\left(\gamma_{e}-1\right)+\frac{m_{p}^{2}}{4 m_{e}^{2}}\left(\gamma_{e}-1\right)^{2}\right]^{1 / 2} .
$$

\section{APPENDIX B}

\section{PHOTON PRODUCTION}

Nonthermal photons are produced by electron synchrotron radiation, electron-electron and electron-nucleon bremsstrahlung, and Compton-scattered CMB, stellar, and X-ray radiation fields. These components are calculated for both primary electrons and secondary electrons and positrons. Neutral pion-decay $\gamma$-rays are also produced in proton-nucleon interactions. We discuss each component in the order mentioned above.

\section{B1. SYNCHROTRON RADIATION}

We define the synchrotron radiation emissivity as $Q_{\text {synch }}=d N_{\text {synch }} / d V d t d E_{\gamma}$. It is calculated from the expression

$$
Q_{\text {synch }}\left(E_{\gamma}, t\right)=\left(\frac{4 \pi \sqrt{3} e^{3} B}{h^{2} \nu m_{e} c^{3}}\right) \int_{0}^{\pi / 2} d \theta \sin ^{2}(\theta) \int_{K_{e, \text { min }}}^{K_{e, \max }} d K_{e} J_{e}\left(K_{e}, t\right) F\left(\nu / \nu_{c}\right),
$$

where $\theta$ is the electron pitch angle, $E_{\gamma}=h \nu$ is the photon energy of frequency $\nu, \nu_{c}=0.42 \gamma_{e}^{2} B_{-7} \sin (\theta)$ in units of Hz, and $J_{e}\left(E_{e}, t\right)$ is the electron intensity (in units of electrons $\left.\mathrm{cm}^{-2} \mathrm{~s}^{-1} \mathrm{sr}^{-1} E_{e}^{-1}\right)$. The function $F\left(\nu / \nu_{c}\right)$ is defined through the expression

$$
F\left(\nu / \nu_{c}\right)=\left(\frac{\nu}{\nu_{c}}\right) \int_{\nu / \nu_{c}}^{\infty} d x K_{5 / 3}(x)
$$

where $K_{5 / 3}$ is the modified Bessel function of order $5 / 3$. The integration is performed numerically with approximate expressions for $F\left(\nu / \nu_{c}\right)$ given by Ginzburg \& Syrovatskii (1965). Electrons are assumed to be isotropically distributed in pitch angle. In addition, we include the effects of free-free absorption of the synchrotron spectra in the source using the absorption coefficient (Ginzburg \& Syrovatskii 1965)

$$
\alpha_{\mathrm{ff}}\left(\mathrm{cm}^{-1}\right)=10^{-2} \frac{n_{\mathrm{ICM}}^{2}}{\nu^{2} T_{e}^{3 / 2}}\left[17.7+\ln \left(\frac{T_{e}^{3 / 2}}{\nu}\right)\right],
$$

where the electron temperature $T_{e}$ is given in kelvins, the ICM particle number density is in units of $\mathrm{cm}^{-3}$, and the frequency $\nu$ is in hertz. 


\section{B2. BREMSSTRAHLUNG RADIATION}

We calculate the electron-electron $(e-e)$ and electron-nucleon $(e-n)$ bremsstrahlung emissivity by the expression

$$
Q_{\mathrm{brem}, e-e, e-n}\left(E_{\gamma}, t\right)=4 \pi n_{\mathrm{ICM}} \Delta_{\mathrm{He}}^{e, n} \int_{K_{e, \min }}^{K_{e, \max }} d K_{e} J_{e}\left(K_{e}, t\right)\left(\frac{d \sigma}{d E_{\gamma}}\right)_{e-e, p},
$$

where we have included the correction factor $\Delta_{\mathrm{He}}^{e, n}$ for the presence of helium. Approximate values for the helium correction factor are $\Delta_{\mathrm{He}}^{e}=1.2$ and $\Delta_{\mathrm{He}}^{n}=1.4$. The expressions for the differential cross sections are adopted from equation (A1) in Haug (1975) for electron-electron interactions and from equation (3BN) in Koch \& Motz (1959) for electron-nucleon interactions.

\section{B3. COMPTON SCATTERING}

We calculate the Compton scattering emissivity using the full Klein-Nishina cross section (Jones 1968; Blumenthal \& Gould 1970) for relativistic electrons $(\gamma \gg 1)$ by the expression

$$
Q_{\text {comp. } j}\left(E_{\gamma}, t\right)=8 \pi r_{0}^{2}\left(m_{e} c^{2}\right)^{2} \int_{0}^{\infty} d E_{t} \frac{n_{j}\left(E_{t}\right)}{E_{t}} \int_{E_{e}^{\text {thresh }}\left(E_{t}\right)}^{\infty} d E_{e} E_{e}^{-2} J_{e}\left(E_{e}, t\right)\left[2 q \ln (q)+(1+2 q)(1-q)+\frac{\left(q \epsilon_{e, t}\right)^{2}(1-q)}{2\left(1+q \epsilon_{e, t}\right)}\right] .
$$

We define

$$
\begin{gathered}
\epsilon_{e, t}=\frac{4 E_{e} E_{t}}{\left(m_{e} c^{2}\right)^{2}}, \\
q=\frac{E_{\gamma}}{\epsilon_{e, t}\left(E_{e}-E_{\gamma}\right)}, \\
E_{e}^{\mathrm{thresh}}\left(E_{t}\right)=\frac{E_{\gamma}}{2}\left(1+\sqrt{1+\frac{\left(m_{e} c^{2}\right)^{2}}{E_{\gamma} E_{t}}}\right), \\
n_{j}\left(E_{t}\right)=\frac{15 U_{j} E_{t}^{2}}{\left(\pi k T_{j}\right)^{4}}\left[\exp \left(\frac{E_{t}}{k T_{j}}\right)-1\right]^{-1} .
\end{gathered}
$$

The energy $E_{t}$ is target photon energy, and $E_{e}^{\text {thresh }}\left(E_{t}\right)$ is the minimum energy required by an electron to scatter a photon from $E_{t}$ to energy $E_{\gamma}$. The temperature, number density, and energy density of the $j$ th photon component is given by $T_{j}, n_{j}$, and $U_{j}$, respectively, from $\S 2.1 .2$.

\section{B4. PION-DECAY $\gamma$-RAYS}

We use the method developed by Dermer (1986b) to calculate the $\pi^{0}$-emissivity from proton-proton collisions. The $\pi^{0}$-emissivity is calculated by

$$
Q_{\pi^{0}}^{p-p}\left(K_{\pi^{0}}, t\right)=4 \pi n_{\mathrm{ICM}} \int_{K_{p}^{\mathrm{thresh}}}^{\infty} d K_{p} J_{p}\left(K_{p}, t\right) \frac{d \sigma\left(K_{\pi^{0}}, K_{p}\right)}{d K_{\pi^{0}}},
$$

where we have defined the neutral pion kinetic energy as $K_{\pi^{0}}$, and the differential cross section for $\pi^{0}$-production is calculated from the cross section given by Dermer (1986b), which uses the isobar model of Stecker (1973) for proton energies less than $3 \mathrm{GeV}$ and the scaling model of Stephens \& Badhwar (1981) for protons of energies greater than $7 \mathrm{GeV}$. A linear combination of the two models is assumed between 3 and $7 \mathrm{GeV}$. The $\gamma$-ray emissivity from $\pi_{0}$-decay is calculated from the expression

$$
Q_{\pi^{0}, \gamma}^{p-p}\left(E_{\gamma}, t\right)=2 \int_{E_{\gamma}+m_{\pi^{0}}^{2} c^{4} / 4 E_{\gamma}}^{\infty} d E_{\pi^{0}} \frac{Q_{\pi^{0}}^{p-p}\left(E_{\pi^{0}}, t\right)}{\sqrt{E_{\pi^{0}}^{2}-m_{\pi^{0}}^{2} c^{4}}} .
$$

Considering collisions with heavy nuclei, Dermer (1986b) has shown that the $\gamma$-ray emissivity is enhanced by the multiplicative factor 1.45. The total $\gamma$-ray emissivity therefore becomes

$$
Q_{\pi^{0}, \gamma}^{\text {total }}=1.45 Q_{\pi^{0}, \gamma}^{p-p} .
$$

\section{REFERENCES}

Abraham, P. B., Brunstein, K. A., \& Cline, T. L. 1966, Phys. Rev., 150, 1088

Arnaud, M., \& Evrard, A. E. 1999, MNRAS, 305, 631

Atoyan, A., \& Dermer, C. D. 2001, Phys. Rev. Lett., 87, 221102
Atoyan, A. M., \& Völk, H. J. 2000, ApJ, 535, 45

Badhwar, G. D., Golden, R. L., \& Stephens, S. A. 1977, Phys. Rev. D, 15, 820

Bagchi, J. 2003, MNRAS, in press 
Baring, M. G., Ellison, D. C., Reynolds, S. P., Grenier, I. A., \& Goret, P. 1999, ApJ, 513, 311

Bell, A. R. 1978, MNRAS, 182, 147

Berezinsky, V. S., Blasi, P., \& Ptuskin, V. S. 1997, ApJ, 487, 529

Berezinskii, V. S., \& Grigor'eva, S. I. 1988, A\&A, 199, 1

Berghöfer, T. W., \& Bowyer, S. 2002, ApJ, 565, L17

Berghöfer, T. W., Bowyer, S., \& Korpela, E. 2000a, ApJ, 535, 615 2000b, ApJ, 545, 695

Berrington, R. C. 2000, Ph.D. thesis, Indiana Univ.

Berrington, R. C., Lugger, P. M., \& Cohn, H. N. 2002, AJ, 123, 2261

Bertschinger, E. 1985, ApJS, 58, 39

Blandford, R. D., \& Ostriker, J. P. 1978, ApJ, 221, L29

Blasi, P. 2000, ApJ, 532, L9

Blasi, P., \& Colafrancesco, S. 1999, Astropart. Phys., 12, 169

Blumenthal, G. R., \& Gould, R. J. 1970, Rev. Mod. Phys., 42, 237

Bonamente, M., Lieu, R., \& Mittaz, J. P. D. 2001, ApJ, 547, L7

Bowyer, S., \& Berghöfer, T. W. 1998, ApJ, 506, 502

Bowyer, S., Korpela, E., \& Berghöfer, T. 2001, ApJ, 548, L135

Bowyer, S., Lampton, M., \& Lieu, R. 1996, Science, 274, 1338

Bridle, A. H., \& Fomalont, E. B. 1976, A\&A, 52, 107

Brunetti, G., Setti, G., Feretti, L., \& Giovannini, G. 2001, MNRAS, 320 , 365

Bryan, G. L., \& Norman, M. L. 1998, ApJ, 495, 80

Clarke, T. E., Kronberg, P. P., \& Böhringer, H. 2001, ApJ, 547, L111

Colafrancesco, S. 2001, in AIP Conf. Proc. 587, Gamma 2001: Gamma-

Ray Astrophysics 2001, ed. S. Ritz, N. Gehrels, \& C. R. Shrader (New York: AIP), 427

Colafrancesco, S., \& Blasi, P. 1998, Astropart. Phys., 9, 227

Colless, M. 1989, MNRAS, 237, 799

Colless, M. \& Dunn, A. M. 1996, ApJ, 458, 435

Commins, E. 1973, Weak Interactions (New York: McGraw-Hill)

Dermer, C. D. 1986a, ApJ, 307, 47 1986b, A\&A, 157, 223

Dermer, C. D., \& Schlickeiser, R. 2002, ApJ, 575, 667

Eilek, J. 1999, in Proc. Workshop Diffuse Thermal and Relativistic Plasma in Galaxy Clusters, ed. H. Böhringer, L. Feretti, P. Schuecker, \& V. K. Kapahi (MPE Rep. 271; Garching: MPI), 71

Ensslin, T. A., \& Biermann, P. L. 1998, A\&A, 330, 90

Ensslin, T. A., Biermann, P. L., Klein, U., \& Kohle, S. 1998, A\&A, 332, 395

Ensslin, T. A., \& Brüggen, M. 2002, MNRAS, 331, 1011

Ensslin, T. A., \& Gopal-Krishna. 2001, A\&A, 366, 26

Ensslin, T. A., Simon, P., Biermann, P. L., Klein, U., Kohle, S., Kronberg, P. P., \& Mack, K.-H. 2001, ApJ, 549, L39

Feretti, L., Brunetti, G., Giovannini, G., Govoni, F., \& Setti, G. 2000, in Constructing the Universe with Clusters of Galaxies, ed. F. Durret \& D Gerbal (Paris: IAP)

Forman, W., Bechtold, J., Blair, W., Giacconi, R., Van Speybroeck, L., \& Jones, C. 1981, ApJ, 243, L133

Fujita, Y., \& Sarazin, C. L. 2001, ApJ, 563, 660

Fusco-Femiano, R., Dal Fiume, D., Feretti, L., Giovannini, G., Grandi, P., Matt, G., Molendi, S., \& Santangelo, A. 1999, ApJ, 513, L21

Fusco-Femiano, R., Dal Fiume, D., Orlandini, M., Brunetti, G., Feretti, L., \& Giovannini, G. 2001, ApJ, 552, L97

Fusco-Femiano, R., Dal Fiume, D., Orlandini, M., De Grandi, S., Molendi, S., Feretti, L., Grandi, P., \& Giovannini, G. 2003a, in ASP Conf. Ser. 301, Matter and Energy in Clusters of Galaxies, ed. S. Bowyer \& C.-Y. Hwang (San Francisco: ASP), in press

Fusco-Femiano, R., Orlandini, M., De Grandi, S., Molendi, S., Feretti, L., Giovannini, G., Bacchi, M., \& Govoni, F. 2003b, A\&A, 398, 441

Fusco-Femiano, R., et al. 2000, ApJ, 534, L7

Gabici, S. \& Blasi, P. 2003, ApJ, 583, 695

Geller, M. J., \& Beers, T. C. 1982, PASP, 94, 421

Ginzburg, V. L., \& Syrovatskii, S. I. 1965, ARA\&A, 3, 297

Giovannini, G., \& Feretti, L. 2000, NewA, 5, 335

Gould, R. J. 1975, ApJ, 196, 689

Govoni, F., Feretti, L., Giovannini, G., Böhringer, H., Reiprich, T. H., \& Murgia, M. 2001, A\&A, 376, 803

Haug, E. 1975, Z. Naturforsch., 30a, 1099

Huba, J. D. 1994, NRL Plasma Formulary (Publ. 6790-94-265; Washington, DC: NRL)

Hwang, C.-Y. 1997, Science, 278, 1917

Jones, F. C. 1968, Phys. Rev., 167, 1159

Kaastra, J. S., Lieu, R., Mittaz, J. P. D., Bleeker, J. A. M., Mewe, R., Colafrancesco, S., \& Lockman, F. J. 1999, ApJ, 519, L119

Kang, H., Rachen, J. P., \& Biermann, P. L. 1997, MNRAS, 286, 257
Kassim, N. E., Clarke, T. E., Ensslin, T. A., Cohen, A. S., \& Neumann, D. M. 2001, ApJ, 559, 785

Kawasaki, W., \& Totani, T. 2002, ApJ, 576, 679

Keshet, U., Waxman, E., Loeb, A., Springel, V., \& Hernquist, L. 2003, ApJ, 585, 128

King, I. R. 1966, AJ, 71, 64

Koch, H. W., \& Motz, J. W. 1959, Rev. Mod. Phys., 31, 920

Large, M. I., Mathewson, D. S., \& Haslam, C. G. T. 1959, Nature, 183, 1663

Liang, H., Dogiel, V. A., \& Birkinshaw, M. 2002, MNRAS, 337, 567

Lieu, R., Bonamente, M., \& Mittaz, J. P. D. 1999a, ApJ, 517, L91

Lieu, R., Ip, W.-H., Axford, W. I., \& Bonamente, M. 1999b, ApJ, 510, L25

Lieu, R., Mittaz, J. P. D., Bowyer, S., Breen, J. O., Lockman, F. J., Murphy, E. M., \& Hwang, C.-Y. 1996a, Science, 274, 1335

Lieu, R., Mittaz, J. P. D., Bowyer, S., Lockman, F. J., Hwang, C.-Y., \& Schmitt, J. H. M. M. 1996b, ApJ, 458, L5

Loeb, A., \& Waxman, E. 2000, Nature, 405, 156

Makino, N., Sasaki, S., \& Suto, Y. 1998, ApJ, 497, 555

Markevitch, M., \& Vikhlinin, A. 2001, ApJ, 563, 95

Meyer, J. P. 1972, A\&AS, 7, 417

Miniati, F. 2002, MNRAS, 337, 199

2003, MNRAS, in press

Miniati, F., Jones, T. W., Kang, H., \& Ryu, D. 2001, ApJ, 562, 233

Miniati, F., Ryu, D., Kang, H., Jones, T. W., Cen, R., \& Ostriker, J. P. 2000, ApJ, 542, 608

Miller, J. A., Larosa, T. N. \& Moore, R. L. 1996, ApJ, 461, 445

Mittaz, J. P. D., Lieu, R., \& Lockman, F. J. 1998, ApJ, 498, L17

Moskalenko, I. V., \& Strong, A. W. 1998, ApJ, 493, 694

Murphy, R. J., Dermer, C. D., \& Ramaty, R. 1987, ApJS, 63, 721

Navarro, J. F., Frenk, C. S., \& White, S. D. M. 1997, ApJ, 490, 493

Norman, C. A., Melrose, D. B., \& Achterberg, A. 1995, ApJ, 454, 60

Oegerle, W. R., \& Hill, J. M. 1994, AJ, 107, 857

. 2001, AJ, 122, 2858

Ohno, H., Takizawa, M., \& Shibata, S. 2002, ApJ, 577, 658

Orth, C. D., \& Buffington, A. 1976, ApJ, 206, 312

Owen, F. N., \& Eilek, J. A. 1998, ApJ, 493, 73

Paolillo, M., Andreon, S., Longo, G., Puddu, E., Gal, R. R., Scaramella, R., Djorgovski, S. G., \& de Carvalho, R. 2001, A\&A, 367, 59

Particle Data Group. 1984, Rev. Mod. Phys., 56, S232

Peebles, P. J. E. 1993, Principles of Physical Cosmology (Princeton: Princeton Univ. Press)

Petrosian, V. 2001, ApJ, 557, 560

Pierce, M. J., \& Berrington, R. C. 2003, ApJ, in press

Ram, M., \& Wang, P. Y. 1971, Phys. Rev. Lett., 26, 476 (erratum 26, 1210)

Reimer, O., Pohl, M., Sreekumar, P., \& Mattox, J. R. 2003, ApJ, 588, 155

Rephaeli, Y. 1979, ApJ, 227, 364

Rephaeli, Y., Gruber, D., \& Blanco, P. 1999, ApJ, 511, L21

Reynolds, S. P. 1996, ApJ, 459, L13

Ricker, P. M. 1998, ApJ, 496, 670

Ricker, P. M., \& Sarazin, C. L. 2001, ApJ, 561, 621

Rybicki, G. B., \& Lightman, A. P. 1979, Radiative Processes in Astrophysics (New York: Wiley)

Sarazin, C. L. 1999, ApJ, 520, 529

Scharf, C. A., \& Mukherjee, R. 2002, ApJ, 580, 154

Schindler, S., \& Müller, E. 1993, A\&A, 272, 137

Schlickeiser, R., Sievers, A., \& Thiemann, H. 1987, A\&A, 182, 21

Skibo, J. G. 1993, Ph.D. thesis, Univ. Maryland

Slee, O. B., Rov, A. L., Murgia, M., Andernach, H., \& Ehle, M. 2001, AJ, 122,1172

Sreekumar, P., et al. 1998, ApJ, 494, 523

Stecker, F. W. 1970, Ap\&SS, 6, 377 1973, ApJ, 185, 499

Stephens, S. A., \& Badhwar, G. D. 1981, Ap\&SS, 76, 213

Sturner, S. J., Skibo, J. G., Dermer, C. D., \& Mattox, J. R. 1997, ApJ, 490, 619

Takizawa, M. 2000, ApJ, 532, 183

Totani, T., \& Kitayama, T. 2000, ApJ, 545, 572

Tribble, P. C. 1993, MNRAS, 263, 31

Valtchanov, I., Murphy, T., Pierre, M., Hunstead, R., \& Lémonon, L. 2002, A\&A, 392, 795

Völk, H. J., Aharonian, F. A., \& Breitschwerdt, D. 1996, Space Sci. Rev., 75,279

Wu, X.-P., \& Chiueh, T. 2001, ApJ, 547, 82

Wu, X.-P., \& Xue, Y.-J. 2000, ApJ, 542, 578 